CERN-TH. 6865/93, HUPAPP-93/3, NEIP 93-004

NSF-ITP-93-96, UTTG-13-93

hepth 9308005

2 August 1993

\title{
PERIODS FOR CALABI-YAU AND LANDAU-GINZBURG VACUA
}

\author{
Per Berglund ${ }^{1,2 \sharp}, \quad$ Philip Candelas ${ }^{2,3}, \quad$ Xenia de la Ossa ${ }^{\sharp}$, Anamaría Font $^{5}$, \\ Tristan HüBsch ${ }^{6 b}$, Dubravka JanČić ${ }^{2}$, Fernando Quevedo ${ }^{4}$ \\ ${ }^{1}$ Institute for Theoretical Physics \\ University of California \\ Santa Barbara \\ CA 93106, USA \\ 2 Theory Group \\ Department of Physics \\ University of Texas \\ Austin, TX 78712, USA \\ ${ }^{3}$ Theory Division \\ CERN \\ CH 1211 Geneva 23 \\ Switzerland \\ ${ }^{4}$ Institut de Physique \\ ${ }^{5}$ Departamento de Física \\ Université de Neuchâtel \\ CH-2000 Neuchâtel \\ Switzerland \\ Universidad Central de Venezuela \\ A.P. 20513, Caracas 1020-A \\ Venezuela \\ ${ }^{6}$ Department of Physics \\ Howard University \\ Washington \\ DC 20059, USA
}

\begin{abstract}
The complete structure of the moduli space of Calabi-Yau manifolds and the associated Landau-Ginzburg theories, and hence also of the corresponding lowenergy effective theory that results from $(2,2)$ superstring compactification, may be determined in terms of certain holomorphic functions called periods. These periods are shown to be readily calculable for a great many such models. We illustrate this by computing the periods explicitly for a number of classes of Calabi-Yau manifolds. We also point out that it is possible to read off from the periods certain important information relating to the mirror manifolds.
\end{abstract}

CERN-TH. 6865/93

2 August 1993

\# After Sept. 15th: Institute for Advanced Study, Olden Lane, Princeton, NJ 08540, USA.

b On leave from the Institute "Rudjer Bošković", Zagreb, Croatia. 


\section{Contents}

1. Preamble

2. Hypersurfaces in Weighted $\mathbb{P}^{N}$

2.1 Generalities

2.2 The fundamental expansion

2.3 Convergence and analytic continuation: small $\varphi_{\alpha}$

2.4 Convergence and analytic continuation: general $\varphi_{\alpha}$

2.5 The remaining periods

3. CICY Manifolds

3.1 Complete intersections in a single projective space

3.2 Calabi-Yau hypersurfaces in products of projective spaces

3.3 A natural conjecture

4. Hypersurfaces with Two Parameters

4.1 Fermat hypersurfaces

4.2 A non-Fermat example

4.3 The Picard-Fuchs equations

5. Other Important Generalizations

5.1 Manifolds with no known mirror

5.2 Twisted moduli

5.3 A new look at the contours

6. Concluding Remarks 


\section{Preamble}

Superstring vacua with $(2,2)$ worldsheet supersymmetry depend on two classes of parameters: the complex structure moduli and the Kähler moduli, which are in a 1-1 correspondence with the $E_{6}$-charged matter fields in the low energy effective theory. The dynamics of these matter fields is determined by the geometry on these two components of the parameter space, the kinetic terms being determined by the metric and the Yukawa couplings by a certain natural third rank tensor. A key feature of the geometry is that both the metric and the Yukawa coupling on each of the two sectors of the moduli space are determined from a single holomorphic object that varies over the moduli space. For the space of complex structures this is the holomorphic 3-form $\Omega$, or equivalently, the vector formed

from its periods, $\varpi_{i} \stackrel{\text { def }}{=} \oint_{\gamma^{i}} \Omega$, with the $\gamma^{i}$ a basis of homology cycles. It is reasonable to think of the $\varpi_{i}$ as the components of $\Omega$ and from these periods much of the structure of the moduli space and of the low energy effective theory is readily available.

In virtue of mirror symmetry the moduli space of the Kähler class parameters of a manifold, $\mathcal{M}$, is identified with the space of complex structures of the mirror manifold, $\mathcal{W}$, of $\mathcal{M}$. Thus whenever the mirror of a given manifold is known, a study of the complex structure periods of the mirror, $\mathcal{W}$, allows one to solve for the structure of the space of Kähler class parameters of $\mathcal{M}$. For most constructions, it is easier to determine the complex structure periods $\varpi_{i}$ and so their Kähler class equivalents are determined via the mirror map.

A procedure of explicitly solving for the structure of the complete moduli space of a given family of vacua, or equivalently of finding both the kinetic terms and the Yukawa couplings of the corresponding low energy theory, factors roughly speaking into two stages. (i) Finding a complete basis of (complex structure) periods $\varpi$ and $\hat{\varpi}$ for the manifold and its mirror and (ii) finding a symplectic basis $\Pi_{i}=m_{i}{ }^{j} \varpi_{j}$, with $m$ a constant matrix, such that the cycles corresponding to $\Pi$ form an integral symplectic homology basis. In this basis, the Kähler potential for the moduli space and the Yukawa couplings for the effective theory are given by the expressions $[1,2]$ :

$$
\begin{aligned}
e^{-K} & =-i \int_{\mathcal{M}} \Omega \wedge \bar{\Omega}=-i \Pi^{T} \Sigma \bar{\Pi} \\
y_{\alpha \beta \gamma} & =\int_{\mathcal{M}} \Omega \wedge \partial_{\alpha} \partial_{\beta} \partial_{\gamma} \Omega=\Pi^{T} \Sigma \partial_{\alpha} \partial_{\beta} \partial_{\gamma} \Pi
\end{aligned}
$$

where $\Sigma$ is the matrix $\left(\begin{array}{rr}0 & \mathbf{1} \\ -\mathbf{1} & 0\end{array}\right)$. Of course, in the cases where a construction of the mirror models is not known, this procedure still provides a description of the complex structure sector of the moduli space and this application is logically independent of mirror symmetry. 
This article addresses step (i) of the process outlined above. We present a widely applicable method of calculating the complex structure periods $\varpi_{i}$ for a large number of Calabi-Yau manifolds and Landau-Ginzburg orbifolds. Through the mirror map, the considerations of this article are useful also for purposes of enumerative geometry. Such applications will, however, be developed elsewhere. There is as yet no general method for implementing step (ii), but worked examples may be found in Refs. [3-7]. More precisely the present work is primarily concerned with two observations: (a) that the periods may be calculated for a wide range of models and (b) that although this fact is logically separate from the existence of mirror symmetry nevertheless it is the case that the periods encode important information about the mirror. Thus it is, for example, that for the class of Calabi-Yau manifolds that can be realised as hypersurfaces in weighted $\mathbb{P}_{4}$ 's the periods are written most naturally in terms of the weights of the mirror manifolds.

Before embarking on generalizations let us first recall the computation of the complex structure periods for a one-parameter family of mirrors of quintic threefolds [3]. These mirror manifolds, $\mathcal{W}$, are defined as the locus, $\mathcal{M} / G$ where $\mathcal{M}$ is the zero set of the polynomial

$$
p(x ; \psi)=\sum_{k=1}^{5} x_{k}^{5}-5 \psi x_{1} x_{2} x_{3} x_{4} x_{5},
$$

and the coordinates $x_{k}$ identified under the action of a group, $G$, which is abstractly $\mathbb{Z}_{5}^{3}$ and which is generated by ${ }^{1}$

$$
\begin{aligned}
& g_{0}=\left(\mathbb{Z}_{5}: 1,0,0,0,4\right), \\
& g_{1}=\left(\mathbb{Z}_{5}: 0,1,0,0,4\right), \\
& g_{2}=\left(\mathbb{Z}_{5}: 0,0,1,0,4\right) .
\end{aligned}
$$

Now $b_{1,1}(\mathcal{M})=b_{2,1}(\mathcal{W})=1$, and $\psi$ parametrizes both the complex structures of $\mathcal{W}$ and the variations of the Kähler class of $\mathcal{M}$; hence the two-fold application of such calculations ${ }^{2}$.

The holomorphic three-form is chosen to be

$$
\Omega(\psi)=5 \psi \frac{5^{3}}{(2 \pi i)^{3}} \frac{x_{5} \mathrm{~d} x_{1} \wedge \mathrm{d} x_{2} \wedge \mathrm{d} x_{3}}{\frac{\partial p(x ; \psi)}{\partial x_{4}}}
$$

with a prefactor chosen to simplify later expressions. The $5^{3}$ corresponds to the order of $G$ and the factors of $2 \pi i$ relate to a residue calculation that follows shortly.

1 We will use the notation $\left(\mathbb{Z}_{k}: r_{1}, r_{2}, r_{3}, r_{4}, r_{5}\right)$ for a $\mathbb{Z}_{k}$ symmetry with the action $\left(x_{1}, x_{2}, x_{3}, x_{4}, x_{5}\right) \rightarrow\left(\omega^{r_{1}} x_{1}, \omega^{r_{2}} x_{2}, \omega^{r_{3}} x_{3}, \omega^{r_{4}} x_{4}, \omega^{r_{5}} x_{5}\right)$, where $\omega^{k}=1$.

2 In fact, $\psi$ also parametrizes a 1-dimensional subspace of the 101-dimensional space of complex structures on $\mathcal{M}$ and its mirror analogue of this 1-dimensional slice in the 101-dimensional space of variations of the Kähler class on $\mathcal{W}$. 
We define also a 'fundamental cycle' $B_{0}$

$$
\begin{aligned}
& B_{0}=\left\{x_{k}\left|x_{5}=1, \quad\right| x_{1}|=| x_{2}|=| x_{3} \mid=\delta\right. \\
& \left.x_{4} \text { given by the solution to } p(x)=0 \text { that tends to zero as } \psi \rightarrow \infty\right\}
\end{aligned}
$$

and a fundamental period

$$
\varpi_{0}(\psi)=\int_{B_{0}} \Omega(\psi)
$$

The evaluation of the fundamental period proceeds by noting that

$$
\begin{aligned}
\varpi_{0}(\psi) & =-5 \psi \frac{1}{(2 \pi i)^{4}} \int_{\gamma_{1} \times \cdots \times \gamma_{4}} \frac{x_{5} \mathrm{~d} x_{1} \mathrm{~d} x_{2} \mathrm{~d} x_{3} \mathrm{~d} x_{4}}{p(x ; \psi)} \\
& =-5 \psi \frac{1}{(2 \pi i)^{5}} \int_{\gamma_{1} \times \cdots \times \gamma_{5}} \frac{\mathrm{d} x_{1} \mathrm{~d} x_{2} \mathrm{~d} x_{3} \mathrm{~d} x_{4} \mathrm{~d} x_{5}}{p(x ; \psi)} .
\end{aligned}
$$

In these relations $\gamma_{i}$ denote the circles $\left|x_{i}\right|=\delta$. The first equality follows by noting that in virtue of the definition of $B_{0}$ the value of $x_{4}$ for which $p$ vanishes lies inside the circle $\gamma_{4}$ for sufficiently large $\psi$ and that the residue of $1 / p$ is $1 / \frac{\partial p}{\partial x_{4}}$. The minus sign reflects a choice of orientation for $B_{0}$ and a factor of $5^{3}$ has been absorbed into the contour by taking this to be $\gamma_{1} \times \cdots \times \gamma_{4}$ rather than $\gamma_{1} \times \cdots \times \gamma_{4} / G$. The second equality follows by noting that on account of the homogeneity properties of the integrand under the scaling $x_{k} \rightarrow \lambda x_{k}$ the integral is, despite appearances, independent of $x_{5}$. We have therefore introduced unity in the guise of $\frac{1}{2 \pi i} \int \frac{d x_{5}}{x_{5}}$. The last expression will be easy to generalize and we may in fact consider the residue (1.7) as the definition of $\varpi_{0}$.

The next maneuver is to expand $1 / p$ in powers of $\psi$

$$
\varpi_{0}(\psi)=\frac{1}{(2 \pi i)^{5}} \sum_{m=0}^{\infty} \frac{1}{(5 \psi)^{m}} \int_{\gamma_{1} \times \cdots \times \gamma_{5}} \frac{d x_{1} d x_{2} d x_{3} d x_{4} d x_{5}}{x_{1} x_{2} x_{3} x_{4} x_{5}} \frac{\left(x_{1}^{5}+x_{2}^{5}+x_{3}^{5}+x_{4}^{5}+x_{5}^{5}\right)^{m}}{\left(x_{1} x_{2} x_{3} x_{4} x_{5}\right)^{m}} .
$$

The integrals may be evaluated by residues. The only terms that contribute are the terms in the expansion of $\left(x_{1}^{5}+\cdots+x_{5}^{5}\right)^{m}$ that cancel the factor of $\left(x_{1} \cdots x_{5}\right)^{m}$ in the denominator. Such terms arise only when $m=5 n$ in which case $\left(x_{1}^{5}+\cdots+x_{5}^{5}\right)^{5 n}$ contains $x_{1}^{5 n} x_{2}^{5 n} x_{3}^{5 n} x_{4}^{5 n} x_{5}^{5 n}$ with coefficient $(5 n) ! /(n !)^{5}$. Thus

$$
\varpi_{0}(\psi)=\sum_{n=0}^{\infty} \frac{(5 n) !}{(n !)^{5}(5 \psi)^{5 n}}, \quad|\psi| \geq 1, \quad 0<\arg (\psi)<\frac{2 \pi}{5} .
$$

The restriction on $\arg (\psi)$ is due to the fact that $\varpi_{0}(\psi)$ has branch points when $\psi^{5}=1$ and we take the cuts to run out radially from these points.

The fundamental period may be continued analytically to the region $|\psi|<1$ with the result that

$$
\varpi_{0}(\psi)=-\frac{1}{5} \sum_{m=1}^{\infty} \frac{\Gamma\left(\frac{m}{5}\right)\left(5 \alpha^{2} \psi\right)^{m}}{\Gamma(m) \Gamma^{4}\left(1-\frac{m}{5}\right)}, \quad|\psi|<1 .
$$


The functions $\varpi_{j}(\psi) \stackrel{\text { def }}{=} \varpi_{0}\left(\alpha^{j} \psi\right)$ are a set of five periods any four of which are linearly independent. Owing to the zeros from $\Gamma\left(1-\frac{m}{5}\right)$ in the denominator, the $\varpi_{j}$ satisfy the single constraint

$$
\sum_{j=1}^{5} \varpi_{j}(\psi)=0 .
$$

Our first observation is that the process outlined above for the simple case of the oneparameter family of mirrors of quintic threefolds admits significant generalizations.

There are large classes of Calabi-Yau and Landau-Ginzburg vacua that have been investigated during the past few years and to which we would like to extend the calculation of the periods. Recently, a complete listing of non-degenerate Landau-Ginzburg potentials leading to $N=2$ string vacua with $c=9$ has been achieved [8-10]. 7,555 (about $\frac{3}{4}$ ) of these models admit a standard geometrical interpretation in terms of hypersurfaces defined from the vanishing of a polynomial in a weighted $\mathbb{P}^{4}$. These models are CalabiYau compactifications and it is for this large class of models that we find the fundamental period in Section 2. Our ability to do this is due to the fact that for all these models, there exists generically a contour $\gamma_{1} \times \gamma_{2} \times \gamma_{3} \times \gamma_{4} \times \gamma_{5}$ as in (1.7). For some $\frac{2}{3}$ of these Calabi-Yau compactifications, the mirror models are known in virtue of the construction of Ref. [11]. This construction applies to deformation classes of hypersurfaces for which a smooth representative may be found corresponding to a polynomial which is the sum of five monomials, that is, the number of monomials is equal to the number of projective coordinates. The prescription for finding the polynomial that corresponds to the mirror of a given model proceeds by transposing the matrix of the exponents of the original polynomial and then forming the quotient by a suitable group ${ }^{3}$. As we will see, the fundamental period for this class of models can be nicely written in terms of the weights of the mirror manifold. This is clearly a reflection of mirror symmetry. Thus, these periods can, in some sense, be thought of as periods for the Kähler class parameters of the mirror manifold. As particular cases of the general expression, a more explicit form of the period for Fermat hypersurfaces is given which allows us to reproduce the known results for one and two moduli examples that have been studied. We also present here a method to find the other periods by analytically continuing the fundamental period and using the geometrical symmetries of the model.

Another class of Calabi-Yau models that has been studied is the class of complete intersection Calabi-Yau (CICY) models [13-16]. These spaces correspond to non-singular intersection of hypersurfaces on products of (weighted) projective spaces. The prototypical

3 The class of Fermat surfaces for which $p=\sum_{i} x_{i}^{a_{i}}$, corresponding to the $A$ series of products of minimal models, are invariant under transposition and the prescription to find the mirrors of these models by orbifoldizing the original manifold [12] is recovered as a particular case. 
example of such a model is provided by the Tian-Yau manifold [17]

$$
\mathbb{P}^{3}\left[\begin{array}{lll}
3 & 0 & 1 \\
0 & 3 & 1
\end{array}\right]
$$

The notation denotes that the manifold is realised in $\mathbb{P}^{3} \times \mathbb{P}^{3}$ by three polynomials $p_{\alpha}$. The columns of the matrix give the degrees of the $p_{\alpha}$ in the variables of each of the projective spaces. More generally, there can be $N$ polynomials $p_{\alpha}$ and $F$ factor spaces such that the total dimension of the factor spaces is $N+3$. In favourable cases, such models correspond to Landau-Ginzburg orbifolds [18-20], and we address the question of finding the periods for these models starting with Section 3.

For complete intersection manifolds, explicit constructions of mirror models are less well known and it is harder to write a general expression for the Kähler class periods explicitly. We proceed by first analyzing two simple classes of CICY manifolds. Those with $N=1$ or $F=1$. The mirror manifolds of the CICY manifolds with $N=1$ are the manifolds studied by Libgober and Teitelbaum [21]. These are the mirrors of the five manifolds

$$
\mathbb{P}^{4}[5], \quad \quad \mathbb{P}^{5}[3,3], \quad \mathbb{P}^{5}[2,4], \quad \mathbb{P}^{6}[2,2,3], \quad \mathbb{P}^{7}[2,2,2,2],
$$

which may be realised by transverse polynomials in a single projective space. These manifolds, which are the simplest one modulus CICY manifolds, are of interest also owing to the fact that the structure of their parameter spaces is somewhat different from the one parameter spaces that have been studied hitherto. We also employ the construction of Batyrev [22] to compute the fundamental periods for the mirrors of the five manifolds that may be realised by a single transverse polynomial in a product of projective spaces

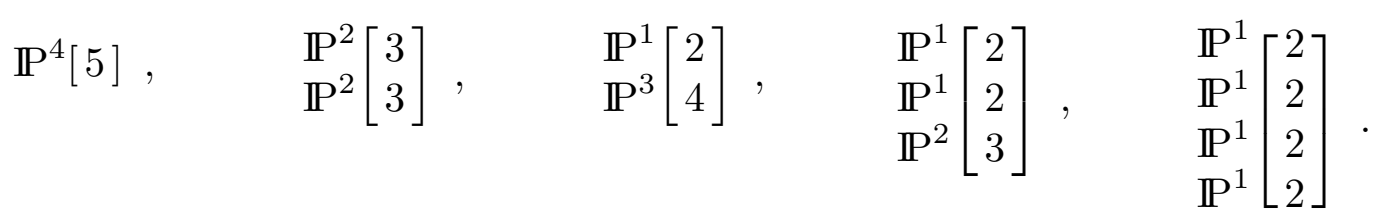

Having computed the fundamental period for these simple CICY manifolds we conjecture the form of the fundamental period for a general CICY and for a general CICY in weighted projective spaces for the Kähler class parameters of the embedding spaces. In Section 4, we study some two-parameter examples to illustrate the analysis.

Section 5 is devoted to other important generalizations. In subsection 5.1 we show that the calculations of Section 2 apply straightforwardly even to Calabi-Yau manifolds which are hypersurfaces in weighted $\mathbb{P}^{4}$ spaces and for which there is not a known construction for its mirror. In subsection 5.2, we point out that the method is not limited to the parameters 
associated to the Kähler-classes of the embedding spaces and as an illustration of this we compute the dependence of the periods on the 'twisted parameters' for the manifolds

$$
\mathbb{P}^{3}\left[\begin{array}{ll}
3 & 1 \\
0 & 3
\end{array}\right] \text { and } \quad \mathbb{P}^{4}\left[\begin{array}{ll}
4 & 1 \\
0 & 2
\end{array}\right]
$$

This is of interest since it requires the introduction of 'non-polynomial' deformations of the defining polynomials. ${ }^{4}$ We find also that it is necessary to introduce a new set of contours in order to evaluate the periods. In connection with this we return in subsection 5.3 to a consideration of the periods for the manifold $\mathbb{P}^{4}[5]$. We introduce a set of alternative contours and argue that these contours are actually the truly fundamental contours in the sense that they seem to exist in general for any Landau-Ginzburg potential associated to a general weighted complete intersection Calabi-Yau manifold. In Section 6, we discuss our results and the limitations of the method.

While completing this work we received a copy of an interesting paper by Batyrev and Van Straten [24] which has substantial overlap with the present work.

4 The first case [23] is of potential phenomenological interest since a particular quotient leads to a three-generation model. Knowledge of the structure of the moduli space will be useful when determining the low-energy effective action for this model. 


\section{Hypersurfaces in Weighted $\mathbb{P}^{N}$}

\subsection{Generalities}

Our aim, in this section, is to derive an explicit expression for the fundamental period for any Calabi-Yau manifold that can be realised as a hypersurface in a weighted $\mathbb{P}^{N}$. We shall show also that this period is most naturally written in terms of data that pertain to the mirror manifold.

We consider a hypersurface $\mathcal{M}$, defined with reference to the zero-set of a defining polynomial $P$ of degree $d$ in a weighted projective space $\mathbb{P}_{\left(k_{0}, \ldots, k_{N}\right)}^{N}$. Of course, the case of immediate interest is $N=4$, but the results of this section are valid for general $N$. The relation

$$
d=\sum_{i=0}^{N} k_{i}, \quad d=\operatorname{deg}(P),
$$

ensures that the hypersurface $\mathcal{M}$ is a Calabi-Yau manifold. In the corresponding LandauGinzburg orbifold with $N+1$ chiral superfields $X_{i}, P$ is the superpotential. It will be shown in later sections how to extend the results to the more general case of (at least many of the) intersections of hypersurfaces in products of projective spaces and the corresponding Landau-Ginzburg orbifolds.

The defining polynomial, $P$, is usually required to be non-degenerate so that the defined hypersurface $\mathcal{M}$ is smooth. That is the derivative, $\mathrm{d} P$, must not vanish simultaneously with $P$ in the (weighted) projective space $\mathbb{P}_{\left(k_{0}, \ldots, k_{N}\right)}^{N}$. In the affine space, $\mathbb{C}_{\left(k_{0}, \ldots, k_{N}\right)}^{N+1}$, $\mathrm{d} P$ and $P$ must simultaneously vanish precisely at the origin $x_{i}=0, i=0, \ldots, N$. This condition seems to be required for the interpretation of Landau-Ginzburg orbifolds: only such non-degenerate models are well understood. The weighted homogeneous coordinates $x_{i}$ have weights $k_{i}$; that is,

$$
x_{i} \approx \lambda^{k_{i}} x_{i}, \quad i=0, \ldots, N .
$$

The basic strategy is to write $P$ as a deformation of a suitable defining polynomial, $P_{0}$, and calculate the periods in terms of a power series expansion in the moduli $\phi_{\alpha}, \alpha=$ $0, \ldots, M-1$, defined by

$$
P\left(x_{i} ; \phi_{\alpha}\right) \stackrel{\text { def }}{=} P_{0}\left(x_{i}\right)-\phi_{0} \prod_{i=0}^{N} x_{i}+\sum_{\alpha=1}^{M-1} \phi_{\alpha} M^{\alpha}\left(x_{i}\right) .
$$

To compare with the simpler case of Ref. [3] recounted in the introduction, write $\phi_{0}=d \psi$. The fundamental period is defined initially for sufficiently large- $\phi_{0}$ and is then extended over the whole parameter space by analytic continuation. 
The fundamental monomial $M^{0}\left(x_{i}\right) \stackrel{\text { def }}{=} \prod_{i} x_{i}$ is a nontrivial deformation at generic points of the moduli space [25], and has here been isolated among other deformations, $M^{\alpha}\left(x_{i}\right)$, for future convenience. In favourable circumstances, the complete list of degree- $d$ monomials $M^{\alpha}\left(x_{i}\right)$, together with the fundamental monomial $\prod_{i} x_{i}$ spans the whole space of deformations. In general, however, there are other, non-polynomial, deformations [26,27] and our present methods seem to imply a restriction to the sector representable by polynomial deformations. It is tempting to identify these deformations with the 'untwisted sector' of Landau-Ginzburg orbifolds or even superconformal field theories, but this is simply false in general [20]; hence the use of the names 'polynomial' and 'non-polynomial' deformations and sectors. We do not have a complete understanding of how to represent in general the parameters of the twisted sectors. However it is sometimes possible to represent certain twisted parameters by certain 'non-polynomial' deformations of the defining polynomial that also involve radicals of polynomials. ${ }^{5}$

For these cases there appears to be no difficulty in calculating periods for these parameters. We present two examples of this procedure in subsection 5.2.

The present methods apply also to finite quotients of Calabi-Yau hypersurfaces since the 'allowable symmetries' leave $\prod_{i} x_{i}$ invariant [29]; the collection of admissible deformations $M^{\alpha}\left(x_{i}\right)$ in this case will of course become smaller. This straightforwardly covers practically all known constructions of mirror models [11] and thereby provides a method for calculating also the Kähler class periods for all these models.

The workhorse of the present article is the residue formula [30,31] for the holomorphic $(N-1)$-form

$$
\Omega\left(\phi_{\alpha}\right) \stackrel{\text { def }}{=} \operatorname{Res}_{\mathcal{M}}\left[\frac{\mu}{P\left(x_{i} ; \phi_{\alpha}\right)}\right],
$$

for the case where each $\mathcal{M}$ (for each choice of $\phi_{\alpha}$ ) is the hypersurface $P\left(x_{i} ; \phi_{\alpha}\right)=0$ in a (weighted) projective space $\mathbb{P}_{\left(k_{0}, \ldots, k_{N}\right)}^{N}$. The residue is evaluated point by point for each $x \in \mathcal{M}$. The resulting $\Omega$ is the holomorphic $(N-1)$-form on the manifold $\mathcal{M}$ parametrized by the $\phi_{\alpha}$. Here,

$$
\mu \stackrel{\text { def }}{=} \frac{1}{(N+1) !} \epsilon^{i_{0} i_{1} \cdots i_{N}} k_{i_{0}} x_{i_{0}} \mathrm{~d} x_{i_{1}} \cdots \mathrm{d} x_{i_{N}}
$$

is the natural holomorphic volume-like form 6 on $\mathbb{P}_{\left(k_{0}, \ldots, k_{N}\right)}^{N}$. Back in the affine space,

5 In certain situations some of the states in the twisted sector of Landau-Ginzburg orbifolds may be represented as rational functions of the coordinates [28]. Thus, one may guess that in general the fractional powers appearing in the 'non-polynomial' defomormations will be negative as well as positive (and zero).

6 Actually, this is not a section of the canonical bundle $\mathcal{K}$ on the weighted projective space, and so is not a holomorphic volume form. Instead, it is a section of the product bundle $\mathcal{K} \otimes \mathcal{O}(d)$. However, the integrand in (2.4) is homogeneous of degree zero and is a section of the canonical 
$\mathbb{C}_{N+1}^{\left(k_{0}, \ldots, k_{N}\right)}$, we may take $\mu$ to be given by

$$
\mu=\prod_{i=0}^{N} \mathrm{~d} x_{i}
$$

Most of the time, the affine case will be implied and the context should make it clear if the projective case is understood instead; we therefore make no notational distinction between these two. The manifold $\mathcal{M}$ is smooth if the defining polynomial $P\left(x_{i} ; \phi_{\alpha}\right)$ is non degenerate and this ensures that the residue (2.4) is well defined.

The fundamental period of $\Omega$ is then defined to be

$$
\varpi_{0}\left(\phi_{\alpha}\right) \stackrel{\text { def }}{=}-\phi_{0} \oint_{B_{0}} \Omega\left(\phi_{\alpha}\right)=-\phi_{0} \frac{C}{(2 \pi i)^{N+1}} \int_{\Gamma} \frac{\prod_{i=0}^{N} \mathrm{~d} x_{i}}{P\left(x_{i} ; \phi_{\alpha}\right)},
$$

where the integral has been re-expressed in the affine space $\mathbb{C}_{N+1}^{\left(k_{0}, \ldots, k_{N}\right)}$ as in Section 1. Here $\Gamma \subset \mathbb{C}_{\left(k_{0}, \ldots, k_{N}\right)}^{N+1}$ is chosen so as to reproduce the integral of the residue (2.4) over the cycle $B_{0}$ in $\mathcal{M}$ and $C$ is a convenient normalization constant. The explicit prefactor $\phi_{0}$ and the overall sign are again merely for later convenience.

\subsection{The fundamental expansion}

Bearing in mind the classification of transverse polynomials of Refs. [11,9,10], we choose the reference polynomial $P_{0}\left(x_{i}\right)$ in Eq. (2.3) to be given by the sum of $N$ monomials

$$
P_{0}\left(x_{i}\right)=\sum_{j=0}^{N} \prod_{i=0}^{N} x_{i}^{a_{i j}}
$$

Thus $P_{0}$ is specified by the $(N+1) \times(N+1)$ matrix of exponents $a_{i j}$ satisfying

$$
\sum_{i=0}^{N} k_{i} a_{i j}=d, \quad \text { for all } j
$$

For $M^{\alpha}\left(x_{i}\right)$ we take the most general set of monomials which we write as

$$
M^{\alpha}=\prod_{i} x_{i}^{q_{i}^{\alpha}} \quad \alpha=1, \cdots, M-1
$$

for some matrix of exponents $q_{i}^{\alpha}$ that is subject to the requirement

$$
\sum_{i=0}^{N} k_{i} q_{i}^{\alpha}=d, \quad \text { for all } \alpha
$$

bundle. This provides for the canonicity of (2.4). 
This covers all the types of non-degenerate polynomials listed in Ref. [11]. For all these, the exponent matrices are block-diagonal, with blocks which are either themselves diagonal or which take one of the following two forms:

$$
\left[\begin{array}{ccccc}
a_{1} & 1 & \cdots & 0 & 0 \\
0 & a_{2} & \cdots & 0 & 0 \\
\vdots & \vdots & \ddots & 1 & 0 \\
0 & 0 & \cdots & a_{r-1} & 1 \\
0 & 0 & \cdots & 0 & a_{r}
\end{array}\right], \quad\left[\begin{array}{ccccc}
a_{1} & 1 & \cdots & 0 & 0 \\
0 & a_{2} & \cdots & 0 & 0 \\
\vdots & \vdots & \ddots & 1 & 0 \\
0 & 0 & \cdots & a_{s-1} & 1 \\
1 & 0 & \cdots & 0 & a_{s}
\end{array}\right]
$$

This class includes, as well as polynomials of Fermat type, also polynomials such as, say,

$$
x_{0}^{7}+x_{1}^{7} x_{3}+x_{3}^{3}+x_{2}^{7} x_{4}+x_{4}^{3},
$$

whose exponent matrix has a $1 \times 1$ Fermat block corresponding to $x_{0}$ and two blocks of the type of the first of the matrices above. The remaining types of non-degenerate polynomials in the complete lists of Refs. $[9,10]$ have more monomials than coordinates. These polynomials are recovered in Eq. (2.3) by including such additional monomials in the collection of $M_{\alpha}\left(x_{i}\right)$. The limit in which the parameter associated to this monomial is zero is however a singular point in the moduli space.

We now turn to the calculation of the fundamental period $\varpi_{0}\left(\phi_{\alpha}\right)$. Rewriting the defining polynomial in Eq. (2.3) as $P=p-\phi_{0} \prod_{i} x_{i}$, we first factor out the fundamental monomial

$$
P=-\phi_{0} \prod_{j} x_{j}\left(1-\frac{p}{\phi_{0} \prod_{i} x_{i}}\right)
$$

and then make a large- $\phi_{0}$ expansion of Eq. (2.7):

$$
\varpi_{0}\left(\phi_{\alpha}\right)=\frac{C}{(2 \pi i)^{N+1}} \oint_{\Gamma} \prod_{j=0}^{N} \frac{\mathrm{d} x_{j}}{x_{j}} \sum_{n=0}^{\infty} \frac{p^{n}}{\phi_{0}^{n} \prod_{i} x_{i}^{n}} .
$$

This is valid regardless of the smoothness (non-degeneracy) of $P_{0}$, since the large- $\phi_{0}$ expansion is equivalent to a small- $\frac{1}{\phi_{0}}$ expansion around the very singular model defined by $P_{\infty}=-\phi_{0} \prod_{i=0}^{N} x_{i}$. At the same time however, the parameters $\phi_{\alpha}, \alpha=0, \ldots, M-1$, parametrize a local neighborhood of the zero-set of $P_{0}$, so the choice of $P_{0}$ defines the $\phi_{\alpha^{-}}$ parametrization. That is, different choices of $P_{0}$ provide different $\phi_{\alpha}$-coordinate patches on the moduli space.

To continue with the calculation started in (2.15), focus now on the expansion of

$$
p^{n}=\left[\sum_{\ell=0}^{N} \prod_{i=0}^{N} x_{i}^{a_{i \ell}}+\sum_{\alpha=1}^{M-1} \phi_{\alpha} \prod_{i=0}^{N} x_{i}^{q_{i}^{\alpha}}\right]^{n} .
$$


Using the multinomial formula,

$$
\left(\sum_{j=1}^{r} X_{j}\right)^{n}=\sum_{\substack{n_{i} \\ \sum n_{i}=n}} \frac{n !}{n_{1} ! n_{2} ! \cdots n_{r} !} X_{1}^{n_{1}} X_{2}^{n_{2}} \cdots X_{r}^{n_{r}}
$$

one finds an explicit expression for $p^{n}$,

$$
p^{n}=\sum_{\substack{n_{i}, m_{\alpha} \\ n_{\ell}+\sum_{\alpha} m_{\alpha}=n}}\left(\frac{n !}{\prod n_{i} ! \prod m_{\alpha} !}\right) \prod_{j=0}^{N} x_{j}^{\left(\sum_{\ell} a_{j \ell} n_{\ell}+\sum_{\alpha} q_{j}^{\alpha} m_{\alpha}\right)}\left(\prod_{\alpha=1}^{M-1} \phi_{\alpha}^{m_{\alpha}}\right) \text {. }
$$

Inserting (2.18) back into the expression (2.15) for $\varpi_{0}\left(\phi_{\alpha}\right)$, each summand contains a product of $N+1$ integrals

$$
\prod_{j=0}^{N} \frac{1}{2 \pi i} \oint \frac{\mathrm{d} x_{j}}{x_{j}} x_{j}^{\left(\sum_{\ell} a_{j \ell} n_{\ell}+\sum_{\alpha} q_{j}^{\alpha} m_{\alpha}-n\right)} .
$$

This $N+1$-fold integral is nonzero precisely if the $N+1$ relations

$$
\sum_{\ell} a_{j \ell} n_{\ell}+\sum_{\alpha} q_{j}^{\alpha} m_{\alpha}=n, \quad j=0, \ldots, N
$$

are satisfied and each integral just picks out the residue at $x_{i}=0$. Multiplying (2.20) by $k_{j}$, summing over $j=0, \ldots, N$ and using Eqs. (2.9), (2.11) and (2.1), we obtain $\sum_{i} n_{i}+\sum_{\alpha} m_{\alpha}=n$, whence the condition imposed on $n_{\ell}, m_{\alpha}$ in the summations of Eq. (2.18) is automatically satisfied for Calabi-Yau hypersurfaces. Thus we find that the fundamental period is then given by by the remarkably simple expression:

$$
\varpi_{0}\left(\phi_{\alpha}\right)=\sum_{n_{i}, m_{\alpha}} \frac{\Gamma(n+1)}{\prod_{\alpha} \Gamma\left(m_{\alpha}+1\right) \prod_{i} \Gamma\left(n_{i}+1\right)} \frac{\prod_{\alpha=1}^{M-1} \phi_{\alpha}^{m_{\alpha}}}{\phi_{0}^{n}}
$$

where

$$
n=\sum_{i} n_{i}+\sum_{\alpha} m_{\alpha}
$$

and the sums over $n_{i}$ and $m_{\alpha}$ are restricted by Eq. (2.20), which provides $N+1$ conditions for $N+M+1$ unknowns $n_{i}, m_{\alpha}, n$. We are left with $M$ free summation variables. There are of course a number of different ways of choosing which variables to sum over. In what follows we will solve for the $n_{i}$.

We now would like to rewrite the above expression for $\varpi_{0}$ in a form which realizes mirror symmetry and which also will turn out to be useful for the analytic continuation to small- $\phi_{0}$ and for obtaining the other periods. The crucial observation here is that 
the summation variables $n_{i}$ and $n$ can be easily eliminated in (2.21), through (2.20) and (2.22). The $n_{i}$ and $n$ may be expressed in terms of the $M$ independent summation variables $\widetilde{m}_{\alpha}, \alpha=1, \ldots, M-1$, and a new variable $r$ by setting

$$
\begin{gathered}
n=\hat{d} r+t \cdot \widetilde{m}, \quad n_{i}=\hat{k}_{i} r+s_{i} \cdot \widetilde{m}, \\
m_{\alpha}=p^{\alpha} \widetilde{m}_{\alpha} \quad(\text { no sum over } \alpha),
\end{gathered}
$$

where the dot-product denotes summation over $\alpha=1, \ldots, M-1$ and where the coefficients $\hat{d}, \hat{k}_{i}, t^{\alpha}$ and $s_{i}{ }^{\alpha}$ satisfy

$$
\begin{aligned}
\sum_{j=0}^{N} \hat{k}_{j} a_{i j} & =\hat{d}, \quad \text { for each } i, \\
\sum_{j=0}^{N} a_{i j} s_{j}{ }^{\alpha}+q_{i}^{\alpha} p^{\alpha} & =t^{\alpha}, \quad \text { for each } i \text { and each } \alpha,
\end{aligned}
$$

for the constraints (2.20) to hold. Note also that (2.22) implies

$$
\hat{d}=\sum_{i=0}^{N} \hat{k}_{i} \quad \text { and } \quad t^{\alpha}=\sum_{i=0}^{N} s_{i}{ }^{\alpha}+p^{\alpha} .
$$

Also, Eqs. (2.23) imply that the new variable $r$ and the quantities $t \cdot \widetilde{m}$ and $s_{i} \cdot \widetilde{m}$, for all $i$, are integers. The $p^{\alpha}$ are chosen so that all $t^{\alpha}$ and $s_{i}^{\alpha}$, and if possible also all $\widetilde{m}_{\alpha}$, are integers; we do not know if this is always possible, but have not been able to find a counterexample.

From these relations we have that

$$
\sum_{j=0}^{N}\left(a_{i j}-1\right) s_{j}^{\alpha}=p^{\alpha}\left(1-q_{i}^{\alpha}\right), \quad \text { for each } i \text { and each } \alpha .
$$

Finally, with the above choice of $a_{i j}$, diagonal or as in (2.12), the matrix $\left[a_{i j}-1\right]$ is invertible in general ${ }^{7}$ and the system of equations (2.27) can be solved for $s_{i}{ }^{\alpha}$ in terms of $p^{\alpha}, q_{i}^{\alpha}$ and $a_{i j}$. The $p^{\alpha}$ may then be chosen so as to obtain integral $s_{i}{ }^{\alpha}$ 's. Thereafter, the $t^{\alpha}$ are determined through (2.26). As promised, Eqs. (2.23) then eliminate the $N+1 n_{i}$ 's and replace $n$ by $r$, leaving $M$ summation variables $r, \tilde{m}_{\alpha}, \alpha=1, \ldots, M-1$.

With these substitutions, the expression for the fundamental period (2.21) becomes

$$
\varpi_{0}\left(\phi_{0}, \phi_{\alpha}\right)=\sum_{r, m_{\alpha}} \frac{\Gamma\left(\hat{d} r+1+\sum_{\alpha} t^{\alpha} m_{\alpha}\right)}{\prod_{i=0}^{N} \Gamma\left(\hat{k}_{i} r+1+\sum_{\alpha} s_{i}{ }^{\alpha} m_{\alpha}\right)} \frac{1}{\phi_{0}^{\hat{d} r}} \prod_{\alpha=1}^{M-1} \frac{\varphi_{\alpha}^{m_{\alpha}}}{\left(p^{\alpha} m_{\alpha}\right) !} ;
$$

7 Amusingly, $\left[a_{i j}-1\right]$ does have one vanishing eigenvalue for $\mathbb{P}^{4}[5]$, owing to $k_{i}=1$, which means that the $s_{i}^{\alpha}$ can be determined only up to a scaling factor per each $\alpha$-which still suffices our present need. 
here and hereafter, we drop the tilde over the $m$ 's. We have also defined the new variables

$$
\varphi_{\alpha} \stackrel{\text { def }}{=}\left(\phi_{\alpha}^{p^{\alpha}} / \phi_{0}^{t^{\alpha}}\right) \text {. }
$$

Equation (2.28) is the main result of this section. It gives an explicit form of the fundamental period for all models defined as transverse hypersurfaces in weighted $\mathbb{P}^{4}$, once we have solved equations (2.24) and (2.25) for the coefficients $\hat{d}, \hat{k}_{i}, t^{\alpha}$ and $s_{i}^{\alpha}$. This is straightforward in all the cases. Most remarkably, upon rewriting the condition (2.24) as $\sum_{j} \hat{k}_{j} a_{j i}^{T}=\hat{d}$, comparison with Eq. (2.9) makes it clear that the $\hat{k}_{i}$ are the weights associated to the transposed polynomial $P_{0}^{T}$ of degree $\hat{d}$ that is used to construct the mirror manifold in the class of models of Ref. [11].

We now illustrate this for the subclass of Fermat hypersurfaces. In this case the matrix $a_{i j}$ is diagonal $a_{i j}=\delta_{i j} d / k_{i}$ and $\hat{d}=d, \hat{k}_{i}=k_{i}$. The independent summation variables are $m_{\alpha}$ and $r$. We find, $s_{i}^{\alpha}=k_{i}\left(t^{\alpha}-q_{i}^{\alpha} p^{\alpha}\right) / d$ and (2.23) become

$$
n=d r+Q, \quad n_{i}=r k_{i}+\frac{k_{i}}{d}\left(Q-Q_{i}\right),
$$

where $Q \equiv m \cdot t^{\alpha}, Q_{i} \equiv p m \cdot q_{i}$. The period then becomes

$$
\varpi_{0}\left(\phi_{0}, \varphi_{\alpha}\right)=\sum_{r, m_{\alpha}} \frac{(d r+Q) ! \prod_{\alpha} \varphi_{\alpha}^{m_{\alpha}}}{\prod_{j=0}^{N}\left(r k_{j}+\frac{k_{j}}{d}\left(Q-Q_{j}\right)\right) ! \prod_{\alpha}\left(p^{\alpha} m_{\alpha} !\left(\phi_{0}\right)^{d r}\right.} .
$$

The case of a single modulus corresponds to setting $M=0$ or equivalently $m_{\alpha}=0$ in Eq. (2.28). In this situation we find that Eq. (2.28) simplifies further to

$$
\varpi_{0}\left(\phi_{0}\right)=\sum_{r} \frac{\Gamma(\hat{d} r+1)}{\prod_{j} \Gamma\left(\hat{k}_{j} r+1\right) \phi_{0}^{\hat{d r}}} .
$$

This agrees with Batyrev's results obtained by means of toric geometry [22]. It is also in agreement with earlier studies restricted to one-modulus models [3-6] and in particular with the quintic discussed in the introduction. Also, from (2.31) we can reproduce the periods for the two moduli Fermat examples studied in Ref. [7]. Applications of Eq. (2.28) in the case of two-parameter models, including non-Fermat hypersurfaces, will be given in Section 4 .

\subsection{Convergence and analytic continuation: small $\varphi_{\alpha}$}

As should be clear from the general expression (2.28), the large- $\phi_{0}$ series expansion of $\varpi_{0}$ will be of the form of a generalized hypergeometric series. The analytic continuation of such series is studied by means of integral representations of Mellin-Barnes type [32,3]. For the cases we consider this provides the analytic continuation of the series (2.28) into the small- $\phi_{0}$ regime. 
The large- $\phi_{0}$ expansion: Let us start by writing (2.28) as

$$
\varpi_{0}\left(\phi_{0}, \varphi_{\alpha}\right)=\sum_{m_{\alpha}} \mathcal{I}_{m}\left(\phi_{0}\right) \frac{\Gamma(t \cdot m+1)}{\prod_{i=0}^{N} \Gamma\left(s_{i} \cdot m+1\right)} \prod_{\alpha=1}^{M-1} \frac{\varphi_{\alpha}^{m_{\alpha}}}{\left(p^{\alpha} m_{\alpha}\right) !}
$$

where

$$
\mathcal{I}_{m}\left(\phi_{0}\right) \stackrel{\text { def }}{=} \sum_{r=0}^{\infty} \phi_{0}^{-\hat{d} r} \frac{(t \cdot m+1)_{\hat{d} r}}{\prod_{i=0}^{N}\left(s_{i} \cdot m+1\right)_{\hat{k}_{i} r}},
$$

and we have used the Pochhammer's symbol

$$
(a)_{r} \stackrel{\text { def }}{=} \frac{\Gamma(a+r)}{\Gamma(a)}
$$

For sufficiently small $\varphi_{\alpha}$, the series $(2.33)$ converges if the function $\mathcal{I}_{m}\left(\phi_{0}\right)$ does, and we now turn to study $\mathcal{I}_{m}\left(\phi_{0}\right)$.

In fact, $\mathcal{I}_{m}\left(\phi_{0}\right)$ is a generalized hypergeometric function ${ }_{\hat{d}+1} F_{\hat{d}}$. To see this, we will need the multiplication formula for the $\Gamma$-function [32](Vol. I):

$$
\Gamma(k z)=(2 \pi)^{\frac{1-k}{2}} k^{k z-\frac{1}{2}} \prod_{q=0}^{k-1} \Gamma\left(z+\frac{q}{k}\right)
$$

whereby

$$
\Gamma(k r+z)=k^{k r} \Gamma(z) \prod_{q=0}^{k-1}\left(\frac{z+q}{k}\right)_{r}, \quad(z)_{k r}=k^{k r} \prod_{q=0}^{k-1}\left(\frac{z+q}{k}\right)_{r}
$$

Thus, we obtain

$$
\begin{aligned}
\mathcal{I}_{m}\left(\phi_{0}\right) & =\sum_{r=0}^{\infty} \frac{z^{-r}}{r !} \frac{\Gamma(r+1) \prod_{p=1}^{\hat{d}}\left(\frac{t \cdot m+p}{\hat{d}}\right)_{r}}{\prod_{i=0}^{N} \prod_{q_{i}=1}^{\hat{k}_{i}}\left(\frac{s_{i} \cdot m+q_{i}}{\hat{k}_{i}}\right)_{r}} \\
& ={ }_{\hat{d}+1} F_{\hat{d}}\left(\begin{array}{l}
a_{1}, \ldots, a_{\hat{d}+1} \\
c_{0}{ }^{1}, \ldots, c_{N}^{\hat{k}_{N}}
\end{array} z^{-1}\right), \quad|z|>1,
\end{aligned}
$$

where

$$
\begin{aligned}
z & =\phi_{0}^{\hat{d}} \frac{\prod_{i=0}^{N} \hat{k}_{i}^{\hat{k}_{i}}}{\hat{d} \hat{d}}, \\
a_{p} & =\frac{t \cdot m+p}{\hat{d}}, \quad p=1, \ldots, \hat{d}, \quad \text { and } a_{\hat{d}+1}=1 \\
c_{i} q^{q_{i}} & =\frac{s_{i} \cdot m+q_{i}}{\hat{k}_{i}}, \quad i=0, \ldots, N, \quad q_{i}=1, \ldots, \hat{k}_{i} .
\end{aligned}
$$


The function $\mathcal{I}_{m}\left(\phi_{0}\right)$ also has a Mellin-Barnes integral representation

$$
\mathcal{I}_{m}\left(\phi_{0}\right)=-\frac{1}{2 \pi i} \frac{\prod_{i=0}^{N} \Gamma\left(s_{i} \cdot m+1\right)}{\Gamma(t \cdot m+1)} \int_{\gamma} \mathrm{d} \rho e^{i \pi \rho} \phi_{0}^{-\hat{d} \rho} \frac{\pi}{\sin \pi \rho} \frac{\Gamma(\hat{d} \rho+t \cdot m+1)}{\prod_{i=0}^{N} \Gamma\left(\hat{k}_{i} \rho+s_{i} \cdot m+1\right)},
$$

where the contour $\gamma$ is parallel to the imaginary axis: $\rho=-\epsilon+i y$, with $\epsilon$ small, real and positive. This integral converges for all $m$ as long as $0<\arg \phi_{0}<\frac{2 \pi}{\hat{d}}$. This can straightforwardly be checked [32](Vol. I, p.49) by using

$$
\lim _{|y| \rightarrow \infty}|\Gamma(x+i y)|=(2 \pi)^{\frac{1}{2}}|y|^{-\frac{1}{2}+x} e^{-\frac{\pi|y|}{2}}, \quad x \text { and } y \text { real },
$$

and taking $\rho=x+i y$, we find that in the limit $|y| \rightarrow \infty$ the integrand in (2.40) behaves as

$$
\left|e^{i \pi \rho} \phi_{0}^{-\hat{d} \rho} \frac{\pi}{\sin \pi \rho} \frac{\Gamma(\hat{d} \rho+t \cdot m+1)}{\prod_{i=0}^{N} \Gamma\left(\hat{k}_{i} \rho+s_{i} \cdot m+1\right)}\right| \sim \frac{\kappa}{2 \pi}|z|^{-x}|y|^{p \cdot m-\frac{N}{2}} e^{-|y|[\pi \pm(\pi-\hat{d} \theta)]}
$$

where we use the notation $(2.39), \theta \stackrel{\text { def }}{=} \arg \phi_{0}$ and

$$
\kappa \stackrel{\text { def }}{=}(2 \pi)^{\frac{4-N}{2}}\left[\frac{\hat{d}^{\frac{1}{2}+t \cdot m}}{\prod_{i=0}^{N} \hat{k}_{i}^{\frac{1}{2}+s_{i} \cdot m}}\right] .
$$

It is easy to see that the integral representation (2.40) reproduces the series expansion for the fundamental period (2.28) by closing the contour of integration in (2.40) to the right with a semicircle of infinite radius and summing over the residues at the poles enclosed by this contour. The only poles that this contour encloses occur at $\rho=r, r=0,1,2, \ldots$, due to the factor $1 / \sin \pi \rho$ in the integrand. It remains to check, however, that the integral over the infinite semicircle vanishes. Using Stirling's formula

$$
\Gamma(z) \sim(2 \pi)^{\frac{1}{2}} e^{-z} z^{z-\frac{1}{2}}
$$

we find that the integrand on the semicircle has the following behaviour as $|\rho| \rightarrow \infty$

$$
\begin{aligned}
\left|e^{i \pi \rho} \phi_{0}^{-\hat{d} \rho} \frac{\pi}{\sin \pi \rho} \frac{\Gamma(\hat{d} \rho+t \cdot m+1)}{\prod_{i=0}^{N} \Gamma\left(\hat{k}_{i} \rho+s_{i} \cdot m+1\right)}\right| & \\
& \sim \frac{\kappa}{2 \pi}|z|^{-|\rho| \mid \cos \chi}|\rho|^{p \cdot m-\frac{N}{2}} e^{-|\rho||\sin \chi|[\pi \pm(\pi-\hat{d} \theta)]},
\end{aligned}
$$

where $\chi=\arg \rho$, with $|\chi|<\frac{\pi}{2}$, and again $0<\theta<2 \pi / \hat{d}$. Thus, the infinite semicircle contribution vanishes for any $m_{\alpha}$ as long as

$$
|z|>1, \quad \text { that is, } \quad\left|\phi_{0}\right|>\hat{d}\left(\prod_{i=0}^{N} \hat{k}_{i}^{\hat{k}_{i}}\right)^{-1 / \hat{d}}
$$


which is precisely the region of convergence of $\mathcal{I}_{m}$ in $(2.38)$.

The small- $\phi_{0}$ expansion: By closing the contour $\gamma$ to the left instead, where $|\chi|>\frac{\pi}{2}$, a series representation for the fundamental period is obtained in the complementary region

$$
|z|<1, \quad \text { that is, } \quad\left|\phi_{0}\right|<\hat{d}\left(\prod_{i=0}^{N} \hat{k}_{i}^{\hat{k}_{i}}\right)^{-1 / \hat{d}} .
$$

In the region now enclosed by the contour, the integrand has

1. poles at $\rho=-(t \cdot m+S) / \hat{d}, S=1,2, \ldots$, from $\Gamma(\hat{d} \rho+t \cdot m+1)$;

2. poles at $\rho=-R, R=1,2, \ldots$, from $1 / \sin \pi \rho$;

3. zeros at $\rho=-\left(s_{i} \cdot m+S_{i}\right) / \hat{k}_{i}, S_{i}=1,2, \ldots$, from $\Gamma\left(\hat{k}_{i} \rho+s_{i} \cdot m+1\right)$, for each $i=0, \ldots, N$. We recall that $t \cdot m, s_{i} \cdot m \in \mathbb{Z}, i=0, \ldots, N$ by virtue of their definition (2.23). Therefore, the poles in item 1 . include all integral $\rho \leq-(t \cdot m+1) / \hat{d}$, which coincide with poles from item 2.; these will then be double poles unless canceled by the zeros from item 3 . Indeed, the zeros in item 3 . include all integral $\rho \leq-\left(s_{i} \cdot m+1\right) / \hat{k}_{i}, i=0, \ldots, N$. Thus, when $\rho \in \mathbb{Z}$ and $\rho \leq-\left(s_{i} \cdot m+1\right) / \hat{k}_{i}$ for one or two $i$ 's, integrand in (2.40) has simple poles or is analytic, respectively, rather than having double poles.

This possible appearance of double poles is in fact a standard characteristic of hypergeometric functions [32] and could be anticipated already from the form (2.38): since $t \cdot m \in \mathbb{Z}$, one of $a_{p}$ in (2.39) must differ from $a_{\hat{d}+1}=1$ by an integer. Similarly, their (partial) cancellation is indicated by some of the $c_{i}{ }^{q_{i}}$ 's being integral - which happens once for every $i=0, \ldots, N$ because $s_{i} \cdot m$ are all integers.

This dependence of the pole structure on the relative values of $t \cdot m$ and $s_{i} \cdot m$ prevents us from writing a general expression in closed form for the small- $\phi_{0}$ expansion of $\varpi_{0}\left(\phi_{0}, \varphi_{\alpha}\right)$. The following brief discussion should help a case by case analysis; recall that Eqs. (2.27) determine the $s_{i}^{\alpha}$, whereupon Eqs. (2.26) determine the $t^{\alpha}$.

From the items 1.-3. above, the following is clear:

For fixed integers $m_{\alpha} \geq 0$ and $R>0$, the extra pole at $\rho=-R$ :

a. remains uncancelled if $\left(1+s_{i} \cdot m-\hat{k}_{i} R\right)>0$ for all $i=0, \ldots, N$, and

b. is a double pole if also $(1+t \cdot m-\hat{d} R) \leq 0$.

The condition a. implies the "sum rule"

$$
\sum_{i=0}^{N} s_{i} \cdot m+N+1>\hat{d} R
$$

for the pole from $1 / \sin \pi \rho$ at $\rho=-R$ to remain. Combined with condition b., this yields

$$
\sum_{i=0}^{N} s_{i} \cdot m+N>t \cdot m, \quad \text { for double poles to occur. }
$$




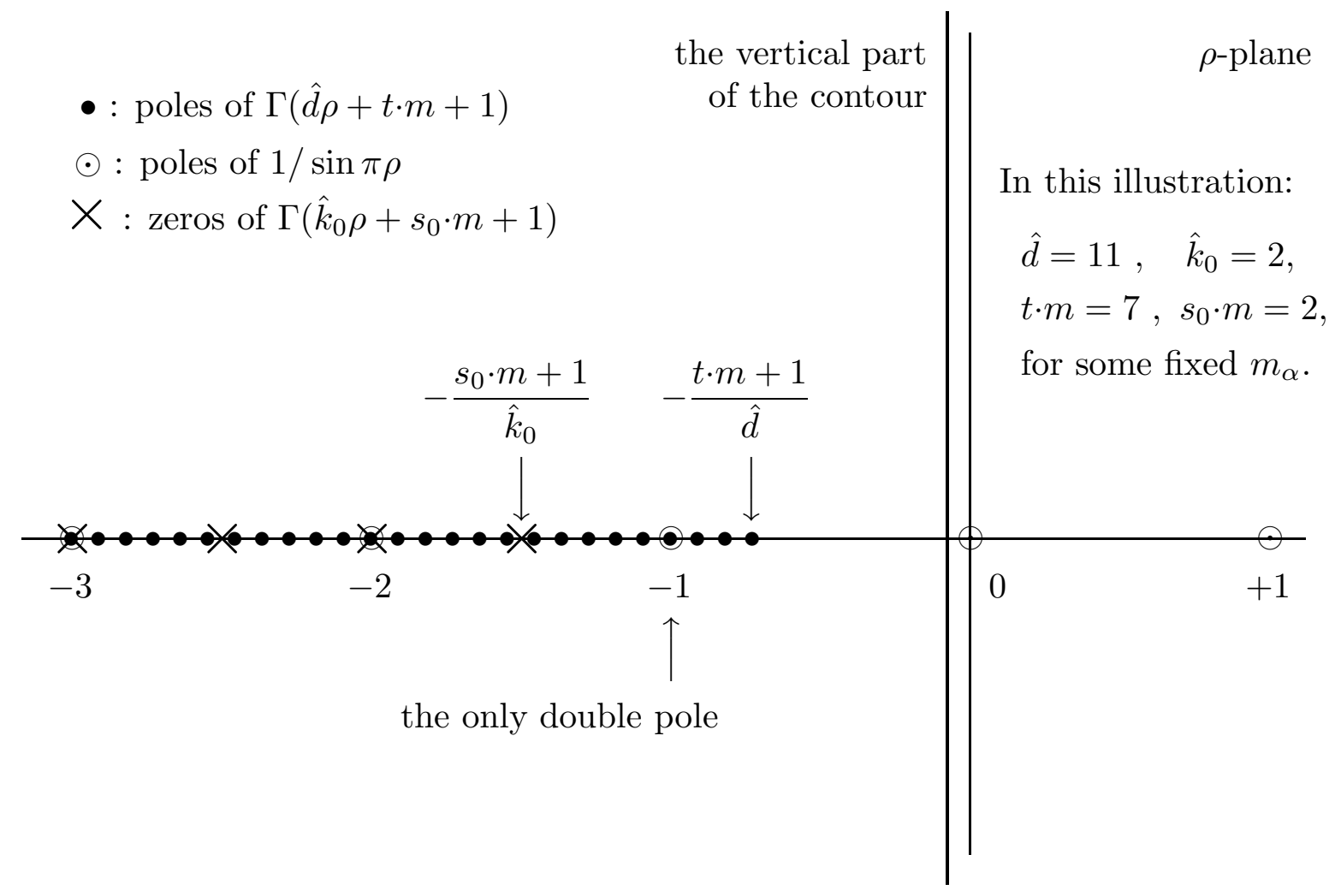

Figure 1. A sketch of a possible pole structure for $\mathcal{I}_{m}\left(\phi_{0}\right)$ in Eq. $(2.40)$.

Finally, using the second of Eqs. (2.26), we obtain

$$
N>p \cdot m, \quad \text { for double poles to occur. }
$$

Therefore, there are at most a few - if any-double poles. Further conditions may be derived from Eqs. (2.23) by recalling that $n, n_{i}, m_{\alpha} \geq 0$, but these do not appear very helpful in the general case.

To describe the small- $\phi_{0}$ evaluation of the integral (2.40), focus first on the nonintegral$\rho$ poles:

$$
\begin{aligned}
\text { simple poles }: & \rho^{\sharp}=-\left(R+\frac{r}{\hat{d}}\right), \quad R=0,1,2 \ldots, \\
r & =t \cdot m, t \cdot m+1, \ldots, t \cdot m+\hat{d}-1, \quad r \neq 0(\bmod \hat{d}),
\end{aligned}
$$

which stem solely from $\Gamma(\hat{d} \rho+t \cdot m+1)$. This 'rational- $\rho$ ' part of $\mathcal{I}_{m}$ is straightforwardly 
obtained

$$
\begin{aligned}
\mathcal{I}_{m}^{(Q)}\left(\phi_{0}\right)=-\frac{(-1)^{p \cdot m}}{\hat{d} \pi^{N}} \frac{\prod_{i=0}^{N} \Gamma\left(s_{i} \cdot m+1\right)}{\Gamma(t \cdot m+1)} & \sum_{\substack{r=t \cdot m \\
r \neq 0(\bmod \hat{d})}}^{t \cdot m+\hat{d}-1} e^{-\frac{i \pi r}{\hat{d}}} \frac{\prod_{j=0}^{N} \sin \left(\frac{\hat{k}_{j} r \pi}{\hat{d}}\right)}{\sin \left(\frac{\pi r}{\hat{d}}\right)}\left(-\phi_{0}\right)^{r} \\
& \times \sum_{R=0}^{\infty} \frac{\prod_{j=0}^{N} \Gamma\left(\hat{k}_{j}\left(R+\frac{r}{\hat{d}}\right)-s_{j} \cdot m\right)}{\Gamma(\hat{d} R+r-t \cdot m)} \phi_{0}^{\hat{d} R} .
\end{aligned}
$$

Correspondingly,

$$
\begin{aligned}
\varpi_{0}^{(Q)}=-\frac{1}{\hat{d} \pi^{N}} \sum_{m_{\alpha}}(-1)^{p \cdot m} & \sum_{\substack{r=t \cdot m \\
r \neq 0(\bmod \hat{d})}}^{t \cdot m+\hat{d}-1} e^{-\frac{i \pi r}{\hat{d}}} \frac{\prod_{j=0}^{N} \sin \left(\frac{\hat{k}_{j} r \pi}{\hat{d}}\right)}{\sin \left(\frac{\pi r}{\hat{d}}\right)}\left(-\phi_{0}\right)^{r} \\
& \times \sum_{R=0}^{\infty} \frac{\prod_{j=0}^{N} \Gamma\left(\hat{k}_{j}\left(R+\frac{r}{\hat{d}}\right)-s_{j} \cdot m\right)}{\Gamma(\hat{d} R+r-t \cdot m)} \phi_{0}^{\hat{d} R} \prod_{\alpha=1}^{M-1} \frac{\varphi_{\alpha}^{m_{\alpha}}}{\left(p^{\alpha} m_{\alpha}\right) !} .
\end{aligned}
$$

Now, to account for the possible integral- $\rho$ poles, let

$$
R_{D} \stackrel{\text { def }}{=}-\left[-\frac{t \cdot m}{\hat{d}}\right]
$$

mark the onset of candidate double poles, where $[x]$ denotes the largest integer not bigger than $x$. Also, let $R_{1}$ and $R_{0}$, respectively, denote the smallest and the second-smallest integer among $\left\{-\left[-\frac{s_{i} \cdot m}{\hat{k}_{i}}\right]\right\}$. These mark, respectively, the onset of the first and the second band of zeros; for $\rho \leq-R_{0}$, all integral- $\rho$ poles are canceled. Clearly, $R_{0} \geq R_{1}$. In addition, assume that

$$
-R_{0} \leq-R_{1} \leq-R_{D} \leq-1
$$

so that the integral- $\rho$ residue contributions to (2.40) come from simple poles for $-R_{0}<$ $\rho \leq-R_{1}$ and $-R_{D}<\rho \leq-1$, but double poles for $-R_{1}<\rho \leq-R_{D}$. Straightforwardly then, we can write

$$
\varpi_{0}\left(\phi_{0}, \varphi_{\alpha}\right)=\varpi_{0}^{(Q)}+\varpi_{0}^{(Z)}, \quad \varpi_{0}^{(Z)}=\varpi_{0}^{(1)}+\varpi_{0}^{(2)}+\varpi_{0}^{(3)},
$$


with

$$
\begin{aligned}
& \varpi_{0}^{(1)}=-\frac{1}{\hat{d}} \sum_{m_{\alpha}} \sum_{R=1}^{R_{D}-1} \frac{\Gamma(1+t \cdot m-\hat{d} R)}{\prod_{j=0}^{N} \Gamma\left(1+s_{j} \cdot m-\hat{k}_{j} R\right)} \phi_{0}^{\hat{d} R} \prod_{\alpha=1}^{M-1} \frac{\varphi_{\alpha}^{m_{\alpha}}}{\left(p^{\alpha} m_{\alpha}\right) !}, \\
& \varpi_{0}^{(2)}=-\frac{1}{\hat{d}} \sum_{m_{\alpha}} \sum_{R=R_{D}}^{R_{1}-1} \frac{(-1)^{t \cdot m+1}\left(-\phi_{0}\right)^{\hat{d} R}}{\Gamma(\hat{d} R-t \cdot m) \prod_{j=0}^{N} \Gamma\left(1+s_{j} \cdot m-\hat{k}_{j} R\right)} \prod_{\alpha=1}^{M-1} \frac{\varphi_{\alpha}^{m_{\alpha}}}{\left(p^{\alpha} m_{\alpha}\right) !} \\
& \times\left[\ln \left[-\phi_{0}^{-\hat{d}}\right]+\hat{d} \Psi(\hat{d} R-t \cdot m)-\sum_{j=0}^{N} \hat{k}_{j} \Psi\left(-\hat{k}_{j} R+s_{j} \cdot m+1\right)\right], \\
& \varpi_{0}^{(3)}=-\frac{1}{\hat{d}} \sum_{m_{\alpha}} \sum_{R=R_{1}}^{R_{0}-1} \frac{\Gamma(1+t \cdot m-\hat{d} R)}{\prod_{j=0}^{N} \Gamma\left(1+s_{j} \cdot m-\hat{k}_{j} R\right)} \phi_{0}^{\hat{d} R} \prod_{\alpha=1}^{M-1} \frac{\varphi_{\alpha}^{m_{\alpha}}}{\left(p^{\alpha} m_{\alpha}\right) !},
\end{aligned}
$$

where $\Psi(z)$ is the logarithmic derivative of the $\Gamma$-function, and $\varpi_{0}^{(Q)}$ was given in (2.53). Clearly, sums with an inverted range equal zero. For example, if $R_{1}=R_{0}$, then $\varpi_{0}^{(3)}=0$.

When there is only one parameter the formula is very simple since, in this case, there are no poles at all at $\rho=-R$. We obtain[33]

$$
\varpi_{0}\left(\phi_{0}\right)=-\frac{1}{\hat{d} \pi^{N}} \sum_{r=1}^{\hat{d}-1} e^{-\frac{\pi i r}{\hat{d}}}\left(-\phi_{0}\right)^{r} \frac{\prod_{j=0}^{N} \sin \frac{\hat{k}_{j} r \pi}{\hat{d}}}{\sin \frac{r \pi}{\hat{d}}} \sum_{R=0}^{\infty} \phi_{0}^{\hat{d} R} \frac{\prod_{j=0}^{N} \Gamma\left(\hat{k}_{j}\left(R+\frac{r}{\hat{d}}\right)\right)}{\Gamma(\hat{d} R+r)},
$$

Using Eqs. (2.37), this can now be written in terms of a sum of generalized hypergeometric functions ${ }_{\hat{d}} F_{\hat{d}-1}$. We obtain [33]

$$
\begin{aligned}
& \varpi_{0}\left(\phi_{0}\right)=-\frac{1}{\hat{d} \pi^{N}} \sum_{r=1}^{\hat{d}-1} e^{-\frac{\pi i r}{\hat{d}}}\left(-\phi_{0}\right)^{r} \frac{\prod_{j=0}^{N} \sin \frac{\hat{k}_{j} r \pi}{\hat{d}} \Gamma\left(\frac{\hat{k}_{j} r}{\hat{d}}\right)}{\sin \frac{r \pi}{\hat{d}} \Gamma(r)} \\
& \times_{\hat{d}} F_{\hat{d}-1}\left(\begin{array}{c}
\frac{r}{\hat{d}}, \ldots, \frac{r}{\hat{d}}, a_{0}^{1}, \ldots, a_{N}^{\hat{k}_{N}-1} \\
\frac{r+1}{\hat{d}}, \frac{r+2}{\hat{d}} \ldots, \frac{r+\hat{d}-1}{\hat{d}}
\end{array} ; z\right),
\end{aligned}
$$

where

$$
a_{i}^{q_{i}}=\left(\frac{r}{\hat{d}}+\frac{q_{i}}{\hat{k}_{i}}\right), \quad i=0, \ldots, N, \quad q_{i}=0, \ldots, \hat{k}_{i}-1 .
$$

So far, through (2.40), we are able to find a series representation for the fundamental period for any value of the modulus $\phi_{0}$. The series converges for sufficiently small values of the remaining moduli $\varphi_{\alpha}$. The radius of convergence however, is determined by the values of these parameters for which the Calabi-Yau manifold becomes singular. It is difficult to provide any detail about this in general and precise information seems to require study on a case by case basis. 


\subsection{Convergence and analytic continuation: general $\varphi_{\alpha}$}

Looking back at Eq. (2.33), it is clear that $\varpi_{0}\left(\phi_{0}, \varphi_{\alpha}\right)$ will converge for small $\varphi_{\alpha}$ if $\mathcal{I}_{m}\left(\phi_{0}\right)$ does not grow too fast as a function of $m_{\alpha}$. For particular cases, it may be even possible to use known results on the behaviour of generalized hypergeometric functions on their parameters. In general, however, this approach does not seem to be fruitful.

We thus rewrite the fundamental period as

$$
\varpi_{0}=\sum_{r} \frac{\phi_{0}^{-\hat{d} r}}{r !} \frac{\Gamma(r+1) \Gamma(\hat{d} r+1)}{\prod_{i=0}^{N} \Gamma\left(\hat{k}_{i} r+1\right)} \mathcal{W}_{r}(\varphi)
$$

where

$$
\mathcal{W}_{r}(\varphi)=\sum_{m_{\alpha}} \frac{(\hat{d} r+1)_{t \cdot m}}{\prod_{i=0}^{N}\left(\hat{k}_{i} r+1\right)_{s_{i} \cdot m}} \prod_{\alpha} \frac{\varphi_{\alpha}^{m_{\alpha}}}{\left(p^{\alpha} m_{\alpha}\right) !} .
$$

The Mellin-Barnes integral representation of this is

$$
\varpi_{0}=-\frac{1}{2 \pi i} \int_{\gamma} \mathrm{d} \rho \phi_{0}^{-\hat{d} \rho} e^{i \pi \rho} \frac{\pi}{\sin \pi \rho} \Gamma(\hat{d} \rho+1) \frac{\mathcal{W}_{\rho}(\varphi)}{\prod_{i=0}^{N} \Gamma\left(\hat{k}_{i} \rho+1\right)},
$$

where the contour $\gamma$ is chosen as before, parallel to the imaginary axis: $\rho=-\epsilon+i y$, with $\epsilon$ small, real and positive. In view of the previous analysis, this integral converges for small $\varphi_{\alpha}$ as long as $0<\arg \phi_{0}<\frac{2 \pi}{\hat{d}}$.

The pole structure of the integrand in (2.65) is of course the same as the one in (2.40). For $\Re e(\rho)>-\epsilon$, the only poles stem from $1 / \sin \pi \rho$ and are located at $\rho=r, r=0,1,2, \ldots$. For $\rho=-1,-2, \ldots$, the poles stem both from $\Gamma(\hat{d} \rho+1)$ and from $1 / \sin \pi \rho$, and are double poles unless canceled by the zeros of $\mathcal{W}_{\rho} / \prod_{i} \Gamma\left(\hat{k}_{i} r+1\right)$. For $\rho=-(\hat{d} R+r)$, with $R=$ $0,1, \ldots$ and $r=1,2, \ldots,(\hat{d}-1), \Gamma(\hat{d} \rho+1)$ has single poles. Indeed, although $\mathcal{W}_{\rho}$ does have poles, the function $\mathcal{W}_{\rho} / \prod_{i} \Gamma\left(\hat{k}_{i} r+1\right)$ has no poles whatsoever. This is easy to see from the fact that the Pochhammer symbol $(a)_{n}$ has no poles for $n>0$ and vanishes when $a=0,-1,-2, \ldots$ but also $n+a>0$. Straightforwardly:

$$
\text { zeros of } \frac{\mathcal{W}_{\rho}}{\prod_{i} \Gamma\left(\hat{k}_{i} r+1\right)}: \begin{cases}\rho=-(Z+1) / \hat{d} & Z=0,1,2, \ldots,(t \cdot m-1), \\ \rho=-\left(Z_{i}+s_{i} \cdot m+1\right) / \hat{k}_{i} & Z_{i}=0,1,2, \ldots,\end{cases}
$$

whence the same pole structure is recovered as in (2.40).

Recall that the discussion in the previous section was done for small $\varphi_{\alpha}$; in fact to zeroth order in $\varphi_{\alpha}$. In particular, the radius of convergence only gives a condition on $\phi_{0}$ which does not depend on the $\varphi_{\alpha}$. In order to find the radius of convergence of the Mellin-Barnes integral for general $\varphi_{\alpha}$ we need to know the asymptotic behaviour of $\left|W_{\rho}\right|$ as $|\rho| \rightarrow \infty$; to close the integral in (2.65) to the left or right we have to make sure that

the integral over the semicircle vanishes as the radius goes to infinity. 
Unfortunately, it is difficult to determine the asymptotic behaviour of $\mathcal{W}_{\rho}(\varphi)$ as $|\rho| \rightarrow$ $\infty$. We note however that the second formula in (2.37), combined with iterates of the identity

$$
(a)_{m+n}=(a+m)_{n}(a)_{m}
$$

allow the representation of $\mathcal{W}_{\rho}(\varphi)$ as

$$
\mathcal{W}_{\rho}\left(\varphi_{\alpha}\right)=\sum_{m_{\alpha}} \prod_{\alpha=1}^{M-1} \frac{\prod_{\ell_{\alpha}=0}^{t^{\alpha}-1}\left(\frac{\hat{d} r+1+\sum_{\beta=1}^{\alpha-1} t^{\beta} m_{\beta}+\ell_{\alpha}}{t^{\alpha}}\right)_{m_{\alpha}}}{\prod_{\ell_{\alpha}=0}^{\alpha} \prod_{i=0}^{N}\left(\frac{\hat{k}_{i} r+1+\sum_{\beta=1}^{\alpha-1} s_{i}^{\beta} m_{\beta}+\ell_{\alpha}}{s_{i}^{\alpha}}\right)_{m_{\alpha}}} \frac{1}{\prod_{l_{\alpha}=1}^{\alpha}\left(\frac{l_{\alpha}}{p^{\alpha}}\right)_{m_{\alpha}}} \frac{\zeta_{\alpha}^{m_{\alpha}}}{m_{\alpha} !}
$$

where

$$
\zeta_{\alpha} \stackrel{\text { def }}{=} \frac{\left(t^{\alpha}\right)^{t^{\alpha}} \varphi_{\alpha}}{\left(p^{\alpha}\right)^{p^{\alpha}} \prod_{i=0}^{N}\left(s_{i}^{\alpha}\right)^{s_{i}^{\alpha}}}
$$

are suitably rescaled variables. The admittedly bewildering representation $(2.68)$ of $\mathcal{W}_{\rho}(\varphi)$ however has the virtue of making it clear that $\mathcal{W}_{\rho}(\varphi)$ is a multiple generalized hypergeometric function. The standard techniques [Luke, I:VII] [34] may thus be developed to study the asymptotic behaviour of $\mathcal{W}_{\rho}(\varphi)$ as $|\rho| \rightarrow \infty$, but this is beyond our present scope. Here, we simply assume that the relevant integrals do converge and forego determining the general, $\varphi_{\alpha}$-dependent radii of convergence for the large- $\phi_{0}$ and small- $\phi_{0}$ expansions.

There being only simple poles at $\rho=r=0,1,2, \ldots$ when the contour in (2.65) is closed to the right, it is straightforward to verify that the integral representation of $\varpi_{0}\left(\phi_{0}, \varphi_{\alpha}\right)$ indeed reproduces the small- $\phi_{0}$ expansion (2.63).

As for $\mathcal{I}_{m}\left(\phi_{0}\right)$, the evaluation by residues of $\varpi_{0}$ in (2.65) may be separated into sums over nonintegral- $\rho$ :

$$
\text { simple poles }: \rho^{\sharp}=-\left(R+\frac{r}{\hat{d}}\right), \quad R=0,1,2 \ldots, \quad r=1,2, \ldots, \hat{d}-1,
$$

and integral- $\rho$ residues. The latter are again finite in number and contain a possible interval of double poles, as indicated in (2.55). The parametrization (2.70) clearly avoids integral- $\rho$, but unlike in (2.51), does not manifest that the poles are canceled out down to $\rho=-(t \cdot m) / \hat{d}$ by the zeros (2.66). In this representation, we have

$$
\begin{aligned}
\varpi_{0}^{(Q)}=-\frac{1}{\hat{d} \pi^{N}} \sum_{r=1}^{\hat{d}-1} e^{-\frac{i \pi r}{\hat{d}}} \frac{\prod_{j=0}^{N} \sin \left(\frac{\hat{k}_{j} r \pi}{\hat{d}}\right)}{\sin \left(\frac{\pi r}{\hat{d}}\right)}\left(-\phi_{0}\right)^{r} \\
\quad \times \sum_{R=0}^{\infty} \frac{\prod_{j=0}^{N} \Gamma\left(\hat{k}_{j}\left(R+\frac{r}{\hat{d}}\right)\right)}{\Gamma(\hat{d} R+r)} \phi_{0}^{\hat{d} R} \mathcal{W}_{-\left(R+\frac{r}{\hat{d}}\right)}(\varphi)
\end{aligned}
$$




$$
\begin{aligned}
& \varpi_{0}^{(1)}=-\frac{1}{\hat{d}} \sum_{R=1}^{R_{D}-1}\left[\frac{\Gamma(1-\hat{d} R) \mathcal{W}_{-R}(\varphi)}{\prod_{j=0}^{N} \Gamma\left(1-\hat{k}_{j} R\right)}\right] \phi_{0}^{\hat{d} R} \\
& \varpi_{0}^{(2)}=+\frac{1}{\hat{d}} \sum_{R=R_{D}}^{R_{1}-1} \frac{\mathcal{W}_{-R}(\varphi)\left(-\phi_{0}\right)^{\hat{d} R}}{\Gamma(\hat{d} R) \prod_{j=0}^{N} \Gamma\left(1-\hat{k}_{j} R\right)} \\
& \times\left[\ln \left[-\phi_{0}^{-\hat{d}}\right]+\hat{d} \Psi(\hat{d} R)-\sum_{j=0}^{N} \hat{k}_{j} \Psi\left(1-\hat{k}_{j} R\right)+w_{-R}(\varphi)\right], \\
& \varpi_{0}^{(3)}=-\frac{1}{\hat{d}} \sum_{R=R_{1}}^{R_{0}-1}\left[\frac{\Gamma(1-\hat{d} R) \mathcal{W}_{-R}(\varphi)}{\prod_{j=0}^{N} \Gamma\left(1-\hat{k}_{j} R\right)}\right] \phi_{0}^{\hat{d} R}
\end{aligned}
$$

where

$$
w_{\rho}(\varphi) \stackrel{\text { def }}{=} \frac{1}{\mathcal{W}_{\rho}(\varphi)} \frac{\mathrm{d}}{\mathrm{d} \rho} \mathcal{W}_{\rho}(\varphi) .
$$

Despite appearances, the quantities in square brackets in (2.72) and (2.74) are finite owing to the zeros (2.66).

\subsection{The remaining periods}

The analytic continuation of the fundamental period obtained from (2.40) very often suffices to find the other periods. Let $\mathcal{M}$ denote the zero set of the defining polynomial (2.3). The $\phi_{\alpha}, \alpha=0, \ldots, M-1$ parametrize the space of complex structures for $\mathcal{M}$ centered around the reference polynomial $P_{0}$. This reference manifold typically enjoys some symmetries and those which act diagonally on the coordinates play a special rôle [12]; denote them by $G_{\mathcal{M}}$. Clearly, any two deformations of the reference manifold which are related by a $G_{\mathcal{M}}$ transformation must be identified. Thus, there is a (lifted) action, $\mathcal{A}$, of $G_{\mathcal{M}}$ on the parameter space, the proper moduli space must be (at least) an $\mathcal{A}$-quotient of the parameter space and the point represented by $P_{0}$ becomes an $\mathcal{A}$-orbifold point. Although the full modular group is in general not known, $\mathcal{A}$ is clearly at least a subgroup and acts on the parameter space

$$
\mathcal{A}: \quad \phi_{\alpha} \longmapsto \lambda_{i}^{a_{\alpha}^{i}} \phi_{\alpha} \quad \alpha=0,1, \ldots, M-1, \quad \lambda^{\hat{d}}=1, \quad i=1, \ldots, r,
$$

where $\mathcal{A} \sim \mathbb{Z}_{d_{1}} \times \ldots \times \mathbb{Z}_{d_{r}}$. In particular we have $d_{1}=\hat{d}$ where $\hat{d}$ is the degree of the transposed polynomial, a quotient of which gives the mirror $\mathcal{W}$ of $\mathcal{M}$. We then define the set of periods $\varpi_{j_{1} \ldots j_{r}}$ as

$$
\varpi_{j_{1} \ldots j_{r}}\left(\phi_{\alpha}\right) \stackrel{\text { def }}{=} \varpi_{0}\left(\lambda_{1}^{j a_{\alpha}^{1}} \phi_{\alpha}, \ldots, \lambda_{r}^{j a_{\alpha}^{r}} \phi_{\alpha}\right) .
$$


Assume first that $\varpi_{0}^{(Z)}=0$; that is, only residues at nonintegral- $\rho$ contribute to the small- $\phi_{0}$ expansion of $\varpi_{0}$. Since $\varpi_{0}^{(Q)}$ does not contain $\hat{d}^{\prime}$ th powers of $\phi_{0}$, it follows that

$$
\sum_{j=1}^{\hat{d}} \varpi_{0}^{(Q)}\left(\lambda_{1}^{j} \phi_{0}, \varphi_{\alpha}\right)=\sum_{j=1}^{\hat{d}} \varpi_{0}\left(\lambda_{1}^{j} \phi_{0}, \varphi_{\alpha}\right)=0 .
$$

Thus, at most $\left(\sum_{i} d_{i}\right)-1$ of the $\sum_{i} d_{i}$ periods are linearly independent. However, if at least one of $\varpi_{0}^{(1)}, \varpi_{0}^{(3)}$ is also nonzero, this no longer holds. Clearly, $\varpi_{0}^{(1)}$ and $\varpi_{0}^{(3)}$ are functions of $\phi_{0}^{\hat{d}}$, rather than $\phi_{0}$ and are consequently invariant under the $\mathbb{Z}_{\hat{d}}$ action $\phi_{0} \mapsto \lambda_{1} \phi_{0}$, with $\lambda_{1}^{\hat{d}}=1$. Then

$$
\sum_{j=1}^{\hat{d}} \varpi_{0}\left(\lambda_{1}^{j} \phi_{0}, \varphi_{\alpha}\right)=\hat{d}\left(\varpi_{0}^{(1)}+\varpi_{0}^{(3)}\right) \neq 0 .
$$

Finally, because of the logarithmic term in $(2.58)$ and (2.73), the presence of $\varpi_{0}^{(2)} \neq 0$, i.e. the contribution of double poles in (2.40) and (2.65), would change the behaviour of $\varpi_{0}\left(\phi_{0}, \varphi_{\alpha}\right)$ substantially.

We again assume that $\varpi_{0}^{(Z)}=0$, so that $\varpi_{0}=\varpi_{0}^{(Q)}$. Given the explicit factors of $\sin \left(\frac{\hat{k}_{j} r \pi}{\hat{d}}\right)$ in Eqs. $(2.53)$ and $(2.71)$, it should be clear that the terms with $\hat{k}_{j} r=0(\bmod \hat{d})$ are also absent. This implies additional conditions on the periods, corresponding to a modular group action

$$
\left(\phi_{0}, \phi_{\alpha}\right) \longmapsto\left(\lambda^{\hat{k}_{j}} \phi_{0}, \lambda^{\hat{k}_{j} t^{\alpha}} \phi_{\alpha}\right) .
$$

Of course, further relations may exist in specific models owing to accidental cancellations.

With this we can now obtain a collection of complex structure periods $\varpi_{j}$ for general Calabi-Yau hypersurfaces in weighted projective spaces and so also for the corresponding Landau-Ginzburg models. The action of $\mathcal{A}$ in Eq. (2.76) produces a complete set of such periods for all models described above. This provides a basis for a complete description of the special geometry of the space of complex structures for these models, and also the spaces of (complexified) Kähler classes for their mirror models. In the subsequent sections, we will attempt various generalizations of these results. 


\section{CICY Manifolds}

We now turn to the calculation of the Kähler class periods for some simple models. One difficulty here will be the identification of suitable parameters and mirror symmetry will be found most useful.

\subsection{Complete intersections in a single projective space}

We will compute here the periods for the Kähler class parameters for the five families of manifolds: $\mathbb{P}^{4}[5], \mathbb{P}^{5}[3,3], \mathbb{P}^{5}[2,4], \mathbb{P}^{6}[2,2,3]$ and $\mathbb{P}^{7}[2,2,2,2]$. Let us begin with the manifold $\mathbb{P}^{5}[3,3]$. Libgober and Teitelbaum [21] seem to have correctly guessed the mirror of this manifold by considering the one parameter family, $\mathcal{M}_{\psi}$, of $\mathbb{P}^{5}[3,3]$ manifolds given by the polynomials

$$
\begin{aligned}
& p_{1}=x_{0}^{3}+x_{1}^{3}+x_{2}^{3}-3 \psi x_{3} x_{4} x_{5}, \\
& p_{2}=x_{3}^{3}+x_{4}^{3}+x_{5}^{3}-3 \psi x_{0} x_{1} x_{2} .
\end{aligned}
$$

Libgober and Teitelbaum observe that $(i)$ there is a group of phase symmetries $H$ such that these polynomials are in fact the most general polynomials whose zero locus is invariant under $H$. (ii) The manifold obtained by resolving $\mathcal{M}_{\psi} / H$ has the values of $b_{1,1}$ and $b_{2,1}$ exchanged relative to $\mathbb{P}^{5}[3,3]$. Moreover, (iii) the numbers, $n_{k}$, of instantons of degree $k$ inferred by calculating the Yukawa coupling yields the correct number in degree one and integral values for all $k$ (the number of instantons has been checked also in degree two).

Underlying much of the present work is the important fact that the holomorphic threeform may be realised as a residue where the manifold of interest is a submanifold in a space of higher dimension. For the manifolds of this section, which are given by $N$ transverse

polynomials $p^{\alpha}, \alpha=1, \ldots, N$ in $\mathbb{P}^{N+3}$, the holomorphic three form can be written as a residue of the $(N+3)$-form

$$
\frac{\epsilon^{A_{1} A_{2} \cdots A_{N+4}} x_{A_{1}} \mathrm{~d} x_{A_{2}} \cdots \mathrm{d} x_{A_{N+4}}}{p_{1} p_{2} \cdots p_{N}}
$$

which is integrated around $N$ circles that enclose the loci $p_{\alpha}=0$. Let $\operatorname{deg}(\alpha)$ denote the degree of $p_{\alpha}$ and note that both the numerator and denominator of the $N+3$-form scale the same way under $x_{A} \rightarrow \lambda x_{A}$ in virtue of the Calabi-Yau condition

$$
\sum_{\alpha=1}^{N} \operatorname{deg}(\alpha)=N+4
$$

In view of the above discussion, we may define a fundamental cycle $B_{0}$ for the mirror of $\mathbb{P}^{5}[3,3]$ in analogy with (1.5). We therefore arrive at the following expression for the 
fundamental period that is the analogue of (1.7)

$$
\varpi_{0}=\frac{1}{(2 \pi i)^{6}} \oint_{\gamma_{0} \times \cdots \times \gamma_{5}} \frac{(3 \psi)^{2} \mathrm{~d} x_{0} \cdots \mathrm{d} x_{5}}{p_{1} p_{2}}
$$

By extracting a factor of $x_{0} \cdots x_{5}$ from the denominator and expanding in inverse powers of $\psi$ we have

$$
\varpi_{0}=\frac{1}{(2 \pi i)^{6}} \int \frac{\mathrm{d} x_{0} \cdots \mathrm{d} x_{5}}{x_{0} \cdots x_{5}} \sum_{k=0}^{\infty} \frac{\left[x_{0}^{3}+x_{1}^{3}+x_{2}^{3}\right]^{k}}{\left(3 \psi x_{3} x_{4} x_{5}\right)^{k}} \sum_{l=0}^{\infty} \frac{\left[x_{3}^{3}+x_{4}^{3}+x_{5}^{3}\right]^{l}}{\left(3 \psi x_{0} x_{1} x_{2}\right)^{l}} .
$$

As in the case of the quintic the only terms that contribute are the terms in the sums that are independent of the $x_{A}$. Such terms arise in the product only when $k=l=3 n$ and the contribution is then $\left(\frac{(3 n) !}{(n !)^{3}}\right)^{2}$. Thus, we find

$$
\varpi_{0}=\sum_{n=0}^{\infty} \frac{((3 n) !)^{2}}{(n !)^{6}(3 \psi)^{6 n}}={ }_{4} F_{3}\left(\frac{1}{3}, \frac{2}{3}, \frac{1}{3}, \frac{2}{3} ; 1,1,1 ; \psi^{-6}\right) .
$$

For the remaining manifolds the computations are analogous. The fundamental period is given by the expression

$$
\varpi_{0}=\frac{\prod_{\alpha=1}^{N}(-\operatorname{deg}(\alpha) \psi)}{(2 \pi i)^{N+4}} \int_{\gamma_{1} \times \cdots \times \gamma_{N+4}} \frac{\mathrm{d} x_{1} \cdots \mathrm{d} x_{N+4}}{p_{1} \cdots p_{N}}
$$

The polynomials together with the resulting periods are displayed in Table 3.1

One finds that the periods of Table 3.1 may be related to standard functions by means of the multiplication formula (2.36). We obtain in this way that the periods are given by generalized hypergeometric functions ${ }_{p} F_{q}$. Recall that these are defined by the series

$$
{ }_{p} F_{q}\left(\begin{array}{l}
a_{1}, \ldots, a_{p} \\
c_{1}, \ldots, c_{q}
\end{array} z\right)=\sum_{k=0}^{\infty} \frac{\left(a_{1}\right)_{k}, \ldots,\left(a_{p}\right)_{k}}{\left(c_{1}\right)_{k}, \ldots,\left(c_{q}\right)_{k}} \frac{z^{k}}{k !}
$$

where $(a)_{r}$ is the Pochhammer's symbol (see Eq. (2.35)). In fact, the periods of Table 3.1 are all of the form

$$
{ }_{4} F_{3}\left(\begin{array}{c}
a_{1}, \ldots, a_{4} \\
1,1,1
\end{array} ;(C \psi)^{-d}\right)
$$

with the $a$-indices $\left(a_{1}, a_{2}, a_{3}, a_{4}\right)$ taking the values shown in the last column of Table 3.1. We see that each polynomial of degree $d$ contributes the indices $\frac{1}{d}, \frac{2}{d}, \cdots, \frac{d-1}{d}$.

We will turn now to the analytic continuation for the fundamental periods of these models. We will mostly concentrate on $\mathbb{P}^{5}[3,3]$ to illustrate the procedure since it is analogous for the other cases. The method is essentially the same as for $\mathbb{P}^{4}[5][3]$. 


\begin{tabular}{|c|c|c|c|}
\hline Manifold & Equations & Period & $a$-Indices \\
\hline $\mathbb{P}^{4}[5]$ & $\begin{aligned} p=x_{1}^{5}+x_{2}^{5}+ & x_{3}^{5}+x_{4}^{5}+x_{5}^{5} \\
& -5 \psi x_{1} x_{2} x_{3} x_{4} x_{5}\end{aligned}$ & $\sum_{n=0}^{\infty} \frac{(5 n) !}{(n !)^{5}\left(5^{5} \psi^{5}\right)^{n}}$ & $\frac{1}{5}, \frac{2}{5}, \frac{3}{5}, \frac{4}{5}$ \\
\hline $\mathbb{P}^{5}[3,3]$ & $\begin{array}{l}p_{1}=x_{1}^{3}+x_{2}^{3}+x_{3}^{3}-3 \psi x_{4} x_{5} x_{6} \\
p_{2}=x_{4}^{3}+x_{5}^{3}+x_{6}^{3}-3 \psi x_{1} x_{2} x_{3}\end{array}$ & $\sum_{n=0}^{\infty} \frac{((3 n) !)^{2}}{(n !)^{6}\left(3^{6} \psi^{6}\right)^{n}}$ & $\frac{1}{3}, \frac{2}{3} ; \frac{1}{3}, \frac{2}{3}$ \\
\hline $\mathbb{P}^{5}[2,4]$ & $\begin{array}{l}p_{1}=x_{1}^{2}+x_{2}^{2}+x_{3}^{2}+x_{4}^{2}-2 \psi x_{5} x_{6} \\
p_{2}=x_{5}^{4}+x_{6}^{4}-4 \psi x_{1} x_{2} x_{3} x_{4}\end{array}$ & $\sum_{n=0}^{\infty} \frac{(2 n) !(4 n) !}{(n !)^{6}\left(2^{8} \psi^{6}\right)^{n}}$ & $\frac{1}{2} ; \frac{1}{4}, \frac{2}{4}, \frac{3}{4}$ \\
\hline $\mathbb{P}^{6}[2,2,3]$ & $\begin{array}{l}p_{1}=x_{1}^{2}+x_{2}^{2}+x_{3}^{2}-2 \psi x_{6} x_{7} \\
p_{2}=x_{4}^{2}+x_{5}^{2}-2 \psi x_{1} x_{2} \\
p_{3}=x_{6}^{3}+x_{7}^{3}-3 \psi x_{3} x_{4} x_{5}\end{array}$ & $\sum_{n=0}^{\infty} \frac{((2 n) !)^{2}(3 n) !}{(n !)^{7}\left(2^{5} 3^{2} \psi^{7}\right)^{n}}$ & $\frac{1}{2} ; \frac{1}{2} ; \frac{1}{3}, \frac{2}{3}$ \\
\hline $\mathbb{P}^{7}[2,2,2,2]$ & $\begin{array}{l}p_{1}=x_{1}^{2}+x_{2}^{2}-2 \psi x_{3} x_{4} \\
p_{2}=x_{3}^{2}+x_{4}^{2}-2 \psi x_{5} x_{6} \\
p_{3}=x_{5}^{2}+x_{6}^{2}-2 \psi x_{7} x_{8} \\
p_{4}=x_{7}^{2}+x_{8}^{2}-2 \psi x_{1} x_{2}\end{array}$ & $\sum_{n=0}^{\infty} \frac{((2 n) !)^{4}}{(n !)^{8}\left(2^{8} \psi^{8}\right)^{n}}$ & $\frac{1}{2} ; \frac{1}{2} ; \frac{1}{2} ; \frac{1}{2}$ \\
\hline
\end{tabular}

Table 3.1: The equations appropriate to the computation of the Kähler class fundamental period, the fundamental period and the $a$ indices for complete intersections in a single projective space.

A very useful tool to understand the analytic continuation as well as the behaviour of solutions near regular singular points is the Riemann symbol for the ${ }_{p} F_{q}$ hypergeometric functions (3.8). For $p=q+1=4$ it is given by

$$
\mathcal{P}\left\{\begin{array}{ccc}
0 & \infty & 1 \\
0 & a_{1} & 0 \\
1-c_{1} & a_{2} & 1 \\
1-c_{2} & a_{3} & 2 \\
1-c_{3} & a_{4} & \sum c-\sum a
\end{array} \quad z\right\}
$$

The Riemann symbol gives the indices of the solutions for the regular singular points of 
the space parametrized by $z$. For the five cases in Table 3.1 we have that

$$
\mathcal{P}\left\{\begin{array}{llll}
0 & \infty & 1 & \\
0 & a_{1} & 0 & \\
0 & a_{2} & 1 & (C \psi)^{-d} \\
0 & a_{3} & 2 & \\
0 & a_{4} & 1 &
\end{array}\right\},
$$

where $\sum a_{i}=2$ for all the models, $C$ is a constant which is 1 for all models except $\mathbb{P}^{5}[3,3]$ where $C=(2 / 3)^{1 / 7}$ and $d=\sum$ degrees. The singularity at $\psi=1 / C$ corresponds to a conifold point and the singularity at $\psi=\infty$ corresponds to the large complex structure limit. For $\mathbb{P}^{4}[5]$, the singularity at $\psi=0$ corresponds to an orbifold point with a $\mathbb{Z}_{5}$ symmetry and the corresponding Calabi-Yau manifold is not singular. For the other cases in this series, this is not the case. For example, for $\mathbb{P}^{5}[3,3]$ the space for $\psi=0$ has a singular curve, actually a singular torus $\mathbb{P}^{2}[3]$.

We will use the information provided by the Riemann symbol to find a complete basis for the periods in the region $|\psi|<1 / C$. This basis, which we denote $F_{k}, k=1, \ldots, 4$, is obtained directly from the Riemann symbol by changing variables from $(C \psi)^{-d}$ to $(C \psi)^{d}$ and exctracting factors of $\psi^{a_{i} d}$. We obtain this way as many independent solutions as there are different indices $a_{i}$. For example, by exctracting a factor of $(C \psi)^{a_{1} d}$ we have a solution

$$
\begin{aligned}
& F_{1}(\psi) \sim(C \psi)^{a_{1} d} \mathcal{P}\left\{\begin{array}{cccc}
0 & \infty & 1 & \\
0 & a_{1} & 0 & \\
a_{2}-a_{1} & a_{1} & 1 & (C \psi)^{d} \\
a_{3}-a_{1} & a_{1} & 2 & \\
a_{4}-a_{1} & a_{1} & 1 &
\end{array}\right\} \\
& =(C \psi)^{a_{1} d}{ }_{4} F_{3}\left(a_{1}, a_{1}, a_{1}, a_{1} ; 1-a_{2}-a_{1}, 1-a_{3}-a_{1}, 1-a_{4}-a_{1} ;(C \psi)^{d}\right) .
\end{aligned}
$$

The repetition of indices in the Riemann symbol for a given regular singular point means that the remaining solutions will have logarithms in the region of convergence around the singular point. If the indices $a_{1}$ and $a_{2}$ are equal, the other linearly independent solution with index $a_{1}$ can be obtained, for example, by considering the derivative of the solution $F_{1}$ with respect to the index $a_{1}$. Except for $\mathbb{P}^{4}[5]$, we do in fact find these logarithmic solutions near $\psi=0$ thus making the modular group intrinsically different from that of $\mathbb{P}^{4}[5]$.

Given the basis $\left\{F_{k}\right\}$, we can find the periods $\varpi_{j}$ as linear combinations

$$
\varpi_{j}=\sum_{k=1}^{4} C_{j k} F_{k} .
$$

Most of the coefficients however can be found by using the symmetries of the model. All these spaces have a "geometrical symmetry" $\mathbb{Z}_{d}$ corresponding to $\psi \rightarrow \alpha \psi, \alpha^{d}=1$. In the region $|C \psi|<1$ we then define

$$
\varpi_{j}(\psi)=\varpi_{0}\left(\alpha^{j} \psi\right), \quad j=0, \ldots, d-1 .
$$


There are, of course, linear relations between these periods since only four of them are linearly independent. Equation (3.14) determines $C_{j k}$, for all $j \neq 0$. The coefficients $C_{0 k}$ can be found from the analytic continuation of the period $\varpi_{0}$ for $|C \psi|>1$ to the region $|C \psi|<1$. The periods $\varpi_{j}(\psi)$ in the region $|C \psi|>1$ can also be obtained by analytic continuation to this region of the representations in (3.13) for $|C \psi|<1$.

Consider now $\mathbb{P}^{5}[3,3]$; there will be two solutions with logarithms in the region $|\psi|<1$ (with $C=1$ ). The solutions without logarithms are

$$
\begin{aligned}
F_{k}(\psi) & =(3 \psi)^{2 k} \frac{\Gamma\left(\frac{k}{3}\right)^{4}}{\Gamma(k)^{2}}{ }_{4} F_{3}(\frac{k}{3}, \frac{k}{3}, \frac{k}{3}, \frac{k}{3} ; \overbrace{\frac{k+1}{3}, \frac{k+2}{3}, \frac{k+1}{3}, \frac{k+2}{3}} ; \psi^{6}), \\
& =(3 \psi)^{2 k} \sum_{n=0}^{\infty}(3 \psi)^{6 n} \frac{\Gamma^{6}\left(n+\frac{k}{3}\right)}{\Gamma^{2}(3 n+k)}, \quad k=1,2,
\end{aligned}
$$

where the overbrace means that an index that is equal to 1 must be deleted. The other two linearly independent solutions can be chosen so that

$$
\begin{aligned}
F_{k+2}(\psi)= & \frac{\mathrm{d}}{\mathrm{d} k} F_{k}(\psi) \\
= & \log (3 \psi)^{2} F_{k} \\
& \quad+2(3 \psi)^{2 k} \sum_{n=0}^{\infty}(3 \psi)^{6 n} \frac{\Gamma^{6}\left(n+\frac{k}{3}\right)}{\Gamma^{2}(3 n+k)}\left[\Psi\left(n+\frac{k}{3}\right)-\Psi(3 n+k)\right], \quad k=1,2 .
\end{aligned}
$$

Let

$$
\varpi_{0}(\psi)=\sum_{k=1}^{2}\left(C_{k} F_{k}+C_{k+2} F_{k+2}\right) .
$$

Since this theory has a geometrical symmetry $\mathbb{Z}_{6}$, we define

$$
\varpi_{j}(\psi)=\varpi_{0}\left(\alpha^{j} \psi\right), \quad \alpha^{6}=1,
$$

where not all six $\varpi_{j}(\psi)$ are linearly independent. We obtain

$$
\varpi_{j}(\psi)=\sum_{k=1}^{2} \alpha^{2 k j}\left[\left(C_{k}+\frac{2 \pi i}{3} j C_{k+2}\right) F_{k}+C_{k+2} F_{k+2}\right],
$$

since

$$
\begin{aligned}
F_{k}\left(\alpha^{j} \psi\right) & =\alpha^{2 k j} F_{k}(\psi), \\
F_{k+2}\left(\alpha^{j} \psi\right) & =\alpha^{2 k j}\left[F_{k+2}(\psi)+\frac{2 \pi i}{3} j F_{k}(\psi)\right], \quad k=1,2 .
\end{aligned}
$$


It is easily seen that, as expected, there are two relations between the six periods:

$$
\begin{gathered}
\varpi_{0}+2 \varpi_{1}+3 \varpi_{2}+2 \varpi_{3}+\varpi_{4}=0 \\
\varpi_{0}+\varpi_{1}+\varpi_{2}-\varpi_{3}-\varpi_{4}-\varpi_{5}=0 .
\end{gathered}
$$

It is worthwhile at this point to contrast these solutions with the periods for $\mathbb{P}^{4}[5]$ (and, in fact with any one modulus model embedded in a weighted $\mathbb{P}^{4}$ ). In the present cases, the monodromy transformation $\mathcal{A}$ around the point $\psi=0$ in the moduli space does not satisfy the relation $\mathcal{A}^{d}=1$ associated to a relation between the periods $\sum_{j=0}^{d-1} \varpi_{j}=0$. This is a consequence of the presence of the logarithms in the solutions. That is, after transport around $\psi=0$ the period $\varpi_{0}$ does not return to itself but it goes to $\varpi_{6}=$ $\varpi_{0}+2\left(\varpi_{3}-\varpi_{0}\right)$. Choosing a basis for the periods

$$
\varpi=\left(\begin{array}{c}
\varpi_{2} \\
\varpi_{1} \\
\varpi_{0} \\
\varpi_{5}
\end{array}\right)
$$

the monodromy of the periods around $\psi=0$ can be found to be

$$
\mathcal{A} \varpi(\psi)=\varpi(\alpha \psi)=a \varpi(\psi),
$$

where

$$
a=\left(\begin{array}{rrrr}
-4 & -3 & -2 & 1 \\
1 & 0 & 0 & 0 \\
0 & 1 & 0 & 0 \\
-8 & -6 & -5 & 2
\end{array}\right)
$$

Returning to the analytic continuation of the periods to the region $|\psi|<1$, we still need to find the constants $C_{k}$. To do this we write the Mellin-Barnes integral for $\varpi_{0}$

$$
\varpi_{0}=\frac{1}{2 \pi i} \int_{\gamma} \mathrm{d} \rho e^{i \pi \rho}(3 \psi)^{-6 \rho} \frac{\Gamma^{2}(3 \rho+1) \Gamma(-\rho)}{\Gamma^{5}(\rho+1)},
$$

which converges for $0<\arg \psi<\frac{2 \pi}{6}$. The contour of integration $\gamma$ is $\Re e(\rho)=-\epsilon$ and $-\infty<\Im m(\rho)<\infty$. This integral has possible poles at

$$
\begin{aligned}
& \text { 1. } \rho=n, \quad n=0,1, \ldots \\
& \text { 2. } \rho=-\frac{n}{3}, \quad n=1,2, \ldots
\end{aligned}
$$

Closing the contour to the right we pick up the poles at $\rho=n, n=0,1, \ldots$, and the sum over residues gives back $\varpi_{0}$ for $|\psi|>1$. Closing the contour to the left, we pick up the 
poles at $\rho=-\frac{n}{3}, n=1,2, \ldots$, which are actually double poles. We obtain

$$
\begin{aligned}
\varpi_{0} & =\frac{1}{9} \sum_{n=1}^{\infty}(3 \psi)^{2 n} \alpha^{-n} \frac{\Gamma\left(\frac{n}{3}\right)}{\Gamma^{2}(n) \Gamma^{5}\left(1-\frac{n}{3}\right)}\left\{\log \left[-(3 \psi)^{-6}\right]-\Psi\left(\frac{n}{3}\right)-5 \Psi\left(1-\frac{n}{3}\right)+6 \Psi(n)\right\}, \\
& =-\frac{3^{\frac{3}{2}}}{(2 \pi)^{5}} \sum_{k=1}^{2} \alpha^{-k}\left\{\left[\frac{\pi}{3}\left(-i+5 \cot \frac{k \pi}{3}\right)\right] F_{k}+F_{k+2}\right\}, \quad|\psi|<1
\end{aligned}
$$

Thus

$$
\begin{aligned}
C_{k} & =-\frac{3^{\frac{1}{2}}}{2^{5} \pi^{4}} \alpha^{-k}\left(-i+5 \cot \frac{k \pi}{3}\right), \\
C_{k+2} & =-\frac{3^{\frac{3}{2}}}{(2 \pi)^{5}} \alpha^{-k}, \quad k=1,2,
\end{aligned}
$$

and

$$
\varpi_{j}(\psi)=-\frac{3^{\frac{3}{2}}}{(2 \pi)^{5}} \sum_{k=1}^{2} \alpha^{k(2 j-1)}\left\{\frac{\pi}{3}\left[(2 j-1) i+5 \cot \frac{k \pi}{3}\right] F_{k}+F_{k+2}\right\}, \quad|\psi|<1 .
$$

The reader may enjoy the straightforward exercise of finding the monodromy of the periods around $\psi=1$ and $\psi=\infty$. Following [3], the periods near $\psi=1$ are given by

$$
\varpi_{j}=-\frac{c_{j}}{2 \pi i}\left(\varpi_{1}-\varpi_{0}\right) \log (\psi-1)+g_{j},
$$

with $g_{j}$ analytic for $|\psi-1|<1$ and where the constants $c_{j}$ are

$$
\left(c_{j}\right)=(1,1,-5,10,-8,-5) \text {. }
$$

The matrix $t$ corresponding to the monodromy $\mathcal{T}$ about $\psi=1$ is therefore

$$
t=\left(\begin{array}{rrrr}
1 & 5 & -5 & 0 \\
0 & 0 & 1 & 0 \\
0 & -1 & 2 & 0 \\
0 & 5 & -5 & 1
\end{array}\right)
$$

The monodromy about $\psi=\infty$ can be easily obtained from the fact that a loop around all the singularities $\left(\psi=\alpha^{j}\right.$ for $j=0, \ldots, 5, \psi=0$ and $\left.\psi=\infty\right)$ is contractible:

$$
\mathbf{1}=t_{\infty}(a t)^{6}
$$

Therefore

$$
t_{\infty}^{-1}=\left(\begin{array}{rrrr}
-4 & -23 & 20 & -1 \\
1 & 5 & -5 & 0 \\
0 & 0 & 1 & 0 \\
-8 & -25 & 14 & 2
\end{array}\right)
$$


A series representation for the periods in the region $|\psi|>1$ can be similarly obtained [3] by considering the following integral representation of $F_{k}$

$$
F_{k}=-\frac{(3 \psi)^{2 k}}{2 \pi i} \int_{\gamma} \mathrm{d} s e^{i \pi s}(3 \psi)^{6 s} \frac{\pi}{\sin \pi s} \frac{\Gamma^{6}\left(s+\frac{k}{3}\right)}{\Gamma^{2}(3 s+k)} \quad k=1,2
$$

but here we will not pursue this matter any further.

As a final comment, we would like to remark that the space $\mathbb{P}^{7}[2,2,2,2]$ is a little different from the others in this series in the sense that its periods for small- $\psi$ behave very differently. The singularity at $\psi=0$ is of the same type as the singularity at $\psi=\infty$ and the reason behind this is that the moduli space for this model has an extra symmetry $\psi \rightarrow 1 / \psi$.

\subsection{Calabi-Yau hypersurfaces in products of projective spaces}

Consider next the five families of CICY manifolds:

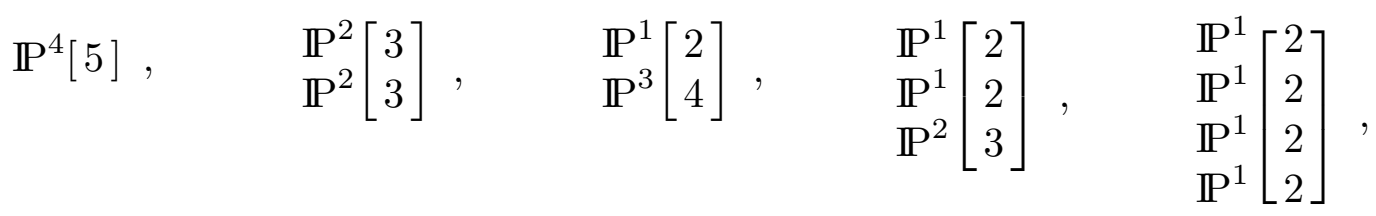

that are defined by a single polynomial. We wish to obtain the fundamental period for their space of Kähler classes using mirror symmetry. Each family has one Kähler class parameter for each $\mathbb{P}^{n}$ factor. The generalization of the analysis presented in Section 2 to these models requires several choices pertaining to the identification of the deformations of the complex structure mirroring the Kähler classes of each $\mathbb{P}^{n}$ factor of the ambient space. We will return to discuss these below. However, for the five families of spaces (3.35) the fundamental period may be found by applying the construction of Batyrev [22].

Consider for example the family of manifolds

$$
\mathbb{P}^{2}\left[\begin{array}{l}
3 \\
3
\end{array}\right]
$$

and denote by $\left(x_{0}, x_{1}, x_{2}\right)$ and $\left(y_{0}, y_{1}, y_{2}\right)$ the homogeneous coordinates of the two $\mathbb{P}^{2}$ 's. We restrict the coordinates to the set $\mathcal{T}$ on which no coordinate vanishes and take $x_{0}=y_{0}=1$ so that $\left(x_{1}, x_{2} ; y_{1}, y_{2}\right)$ are affine coordinates on the embedding space. Let

$$
f=\frac{p}{x_{1} x_{2} y_{1} y_{2}}=\sum_{m_{1}, m_{2} ; n_{1}, n_{2}} a_{m_{1} m_{2} n_{1} n_{2}} x_{1}^{m_{1}} x_{2}^{m_{2}} y_{1}^{n_{1}} y_{2}^{n_{2}} .
$$

The Newton polyhedron, $\Delta$, of the manifold is the convex hull of the points $\left(m_{1}, m_{2} ; n_{1}, n_{2}\right)$ for which the coefficients $a$ of the Laurent polynomial $f$ are nonzero. For this elementary 
example these are the points for which

$$
\begin{gathered}
-1 \leq m_{1}, \quad-1 \leq m_{2} ; \quad-1 \leq n_{1}, \quad-1 \leq n_{2} \\
m_{1}+m_{2} \leq 1 \quad ; \quad n_{1}+n_{2} \leq 1 .
\end{gathered}
$$

The vertices of the polyhedron dual to $\Delta$ are the six points:

$$
\begin{array}{cl}
(1,0,0,0), \quad(0,1,0,0), & (0,0,1,0), \quad(0,0,0,1), \\
(-1,-1,0,0), & (0,0,-1,-1) .
\end{array}
$$

These points are the coefficients in the inequalities (3.38) if the inequalities are replaced by equalities and we multiply through if necessary so that the constant term is -1 in each case. The Laurent polynomial corresponding to the dual polyhedron is

$$
\hat{f}=1+X_{1}+X_{2}+\frac{\lambda_{1}}{X_{1} X_{2}}+Y_{1}+Y_{2}+\frac{\lambda_{2}}{Y_{1} Y_{2}}
$$

where the freedom to make linear redefinitions of the coordinates and to multiply $\hat{f}$ by an overall factor has been used to reduce the number of free parameters to two which is the correct number since $b_{1,1}=2$ for the original manifold (3.36).

We may now revert to homogeneous coordinates by setting $X_{1}=\frac{x_{1}}{x_{0}}, X_{2}=\frac{x_{2}}{x_{0}}$ etc. Then the mirror manifold is given by the singular bicubic hypersurface $\hat{p}=0$ in $\mathbb{P}^{2} \times \mathbb{P}^{2}$ with

$$
\begin{aligned}
\hat{p} & =X_{1} X_{2} Y_{1} Y_{2} \hat{f} \\
& =x_{0} x_{1} x_{2} y_{0} y_{1} y_{2}+\left(x_{1}^{2} x_{2}+x_{1} x_{2}^{2}+\lambda_{1} x_{0}^{3}\right) y_{0} y_{1} y_{2}+\left(y_{1}^{2} y_{2}+y_{1} y_{2}^{2}+\lambda_{2} y_{0}^{3}\right) x_{0} x_{1} x_{2} .
\end{aligned}
$$

The fundamental period for the mirror now follows in a manner that has become familiar:

$$
\begin{aligned}
\varpi_{0}\left(\lambda_{1}, \lambda_{2}\right) & =\frac{1}{(2 \pi i)^{6}} \int \frac{\mathrm{d} x_{0} \mathrm{~d} x_{1} \mathrm{~d} x_{2} \mathrm{~d} y_{0} \mathrm{~d} y_{1} d y_{2}}{\hat{p}} \\
& =\sum_{n_{1}, n_{2}=0}^{\infty} \frac{\left(3 n_{1}+3 n_{2}\right) !}{\left(n_{1} !\right)^{3}\left(n_{2} !\right)^{3}} \lambda_{1}^{n_{1}} \lambda_{2}^{n_{2}} .
\end{aligned}
$$

The other cases follow in precise analogy and we list the fundamental Kähler class periods in Table 3.2 to reveal the pattern. These spaces would seem to furnish rather simple examples of multiparameter spaces which might be easier to analyse than the multiparameter cases studied thus far [4-7]. Note also that these spaces do not have a single 'fundamental' parameter; rather they have several, one corresponding to the Kähler class of each factor space. 


\begin{tabular}{|c|c|}
\hline Manifold & Period \\
\hline $\mathbb{P}^{4}[5]$ & $\sum_{n=0}^{\infty} \frac{(5 n) !}{(n !)^{5}} \lambda^{n}$ \\
\hline $\mathbb{P}^{2}\left[\begin{array}{l}3 \\
3\end{array}\right]$ & $\sum_{n_{1}, n_{2}=0}^{\infty} \frac{\left(3 n_{1}+3 n_{2}\right) !}{\left(n_{1} !\right)^{3}\left(n_{2} !\right)^{3}} \lambda_{1}^{n_{1}} \lambda_{2}^{n_{2}}$ \\
\hline $\mathbb{P}^{1}\left[\begin{array}{l}2 \\
4\end{array}\right]$ & $\sum_{n_{1}, n_{2}=0}^{\infty} \frac{\left(2 n_{1}+4 n_{2}\right) !}{\left(n_{1} !\right)^{2}\left(n_{2} !\right)^{4}} \lambda_{1}^{n_{1}} \lambda_{2}^{n_{2}}$ \\
\hline 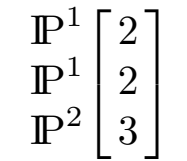 & $\sum_{n_{1}, n_{2}, n_{3}=0}^{\infty} \frac{\left(2 n_{1}+2 n_{2}+3 n_{3}\right) !}{\left(n_{1} !\right)^{2}\left(n_{2} !\right)^{2}\left(n_{3} !\right)^{3}} \lambda_{1}^{n_{1}} \lambda_{2}^{n_{2}} \lambda_{3}^{n_{3}}$ \\
\hline $\left.\begin{array}{l}\mathbb{P}^{1} \\
\mathbb{P}^{1} \\
\mathbb{P}^{1} \\
\mathbb{P}^{1} \\
2 \\
2 \\
2\end{array}\right]$ & $\sum_{n_{1}, n_{2}, n_{3}, n_{4}=0}^{\infty} \frac{\left(2 n_{1}+2 n_{2}+2 n_{3}+2 n_{4}\right) !}{\left(n_{1} !\right)^{2}\left(n_{2} !\right)^{2}\left(n_{3} !\right)^{2}\left(n_{4} !\right)^{2}} \lambda_{1}^{n_{1}} \lambda_{2}^{n_{2}} \lambda_{3}^{n_{3}} \lambda_{4}^{n_{4}}$ \\
\hline
\end{tabular}

Table 3.2: The Kähler class fundamental periods for Calabi-Yau hypersurfaces in products of projective spaces.

\subsection{A natural conjecture}

We now know the Kähler class for the fundamental periods for CICY manifolds for which the degree matrix consists either of a single row or a single column. Based on this, it is natural to conjecture a form for a general weighted CICY - with $F>1$ factor spaces

$$
\mathbb{P}_{\left(k_{1}^{(1)}, \ldots, k_{n_{1}+1}^{(1)}\right)}^{n_{1}} \times \cdots \times \mathbb{P}_{\left(k_{1}^{(F)}, \ldots, k_{n_{F}+1}^{(F)}\right)}^{n_{F}},
$$

and $N>1$ polynomials $p_{\alpha}, \alpha=1, \ldots, N$ such that the degree of $p_{\alpha}$ in the variables of the $j$ 'th projective space is $\operatorname{deg}_{j}(\alpha)$ which, in order to have a Calabi-Yau manifold, they must satisfy the relations

$$
\sum_{\alpha} \operatorname{deg}_{j}(\alpha)=\sum_{i=1}^{n_{j}+1} k_{i}^{(j)}, \quad \text { for all } j .
$$


The fundamental period is then given, as a function of the parameters $\lambda_{j}$ corresponding to the Kähler classes of each factor space, by

$$
\varpi_{0}\left(\lambda_{1}, \ldots, \lambda_{F}\right)=\sum_{m_{1}, \ldots, m_{F}=0}^{\infty} \frac{\prod_{\alpha=1}^{N}\left(\sum_{j=1}^{F} \operatorname{deg}_{j}(\alpha) m_{j}\right) !}{\left(\prod_{i=1}^{n_{1}+1}\left(k_{i}^{(1)} m_{1}\right) !\right) \cdots\left(\prod_{i=1}^{n_{F}+1}\left(k_{i}^{(F)} m_{F}\right) !\right)} \lambda_{1}^{m_{1}} \cdots \lambda_{F}^{m_{F}}
$$

We illustrate this conjecture below. It is compatible with all other examples for which the period has already been calculated $[35,24]$.

By way of an example of this conjecture, consider the Schimmrigk manifold [23],

$$
\mathcal{M} \in \mathbb{P}^{2}\left[\begin{array}{ll}
3 & 1 \\
0 & 3
\end{array}\right]:\left\{\begin{array}{c}
p_{1,0}(x)=\sum_{i=0}^{3} x_{i}^{3}=0 \\
p_{2,0}(x, y)=\sum_{i=1}^{3} x_{i} y_{i}^{3}=0
\end{array}\right.
$$

Identify first $x_{1} x_{2} x_{3}$ and $x_{0} y_{1} y_{2} y_{3}$ as the fundamental deformations of $p_{0}^{1}$ and $p_{0}^{2}$ respectively. Thus, we want to find the periods associated to a deformation of $\mathcal{M}$ given by

$$
\begin{aligned}
& p_{1}=p_{1,0}-\psi_{1} x_{1} x_{2} x_{3}, \\
& p_{2}=p_{2,0}-\psi_{2} x_{0} y_{1} y_{2} y_{3} .
\end{aligned}
$$

Note that $x_{1} x_{2} x_{3}$ and $x_{0} y_{1} y_{2} y_{3}$ are left invariant under action of the quotient symmetry $H$ which produces the mirror manifold, $\mathcal{W}=\mathcal{M} / H$. Therefore, by mirror symmetry, the fundamental period may also be thought of as representing deformations of the Kähler class of $\mathcal{M}$. The period follows straightforwardly as in previous examples,

$$
\varpi_{0}=\left(-\psi_{1}\right)\left(-\psi_{2}\right) \frac{1}{(2 \pi i)^{7}} \oint_{\Gamma} \frac{\mathrm{d} x_{0} \ldots \mathrm{d} y_{3}}{p_{1} p_{2}}
$$

which becomes

$$
\begin{aligned}
\varpi_{0} & =\frac{1}{(2 \pi i)^{7}} \int_{\Gamma} \frac{\mathrm{d} x_{0} \ldots \mathrm{d} y_{3}}{x_{0} \ldots y_{3}} \sum_{n=0}^{\infty}\left(\frac{x_{0}^{3}+\ldots+x_{3}^{3}}{\psi_{1} x_{1} x_{2} x_{3}}\right) \sum_{m=0}^{\infty}\left(\frac{x_{1} y_{1}^{3}+\ldots+x_{3} y_{3}^{3}}{\psi_{2} x_{0} y_{1} y_{2} y_{3}}\right) \\
& =\sum_{m_{1}, m_{2}=0}^{\infty} \frac{\left(3 m_{1}\right) !\left(3 m_{2}+m_{1}\right) !}{\left(m_{1} !\right)^{4}\left(m_{2} !\right)^{3}} \frac{1}{\left(\psi_{1}\right)^{3 m_{2}+m_{1}}\left(\psi_{2}\right)^{3 m_{1}}} .
\end{aligned}
$$

Indeed, upon identifying $\lambda_{1}^{-1}=\psi_{1} \psi_{2}^{3}$ and $\lambda_{2}^{-1}=\psi_{1}^{3}$, this is seen to be the appropriate special case of the conjecture (3.45). Note also that this is not the complete answer; $b_{1,1}=8$ for this CICY manifold while we have here obtained the fundamental period only 
as a function of two moduli, the ones corresponding to the Kähler classes on $\mathbb{P}^{3}$ and $\mathbb{P}^{2}$. The dependence on the remaining six parameters will be discussed in Section 5 .

Another interesting example of the above is to consider the two-parameter (Fermat) models which will be discussed in Section 4.1. For the first four models in (4.1) one can (for specific values of the moduli) relate them to one-parameter families of complete intersections in a weighted projective space. In particular, let us consider a two-parameter deformation of $\mathbb{P}_{(1,1,2,2,6)}^{4}[12]_{-252}^{128,2}$ given by (4.2). From the discussion in Section 4.1 one finds that the fundamental period may be written as

$$
\varpi_{0}=\sum_{l=0}^{\infty} \frac{\Gamma(6 l+1)}{\Gamma^{3}(l+1) \Gamma(3 l+1)}\left(\frac{-1}{(12 \psi)^{6}}\right) U_{l}(\phi)
$$

where $U_{l}(\phi)$ is given by (4.7). At the singular curve $\phi=1, U_{l}(\phi=1)=\frac{\Gamma(2 l+1)}{\Gamma^{2}(l+1)}$, and hence (3.50) becomes

$$
\varpi_{0}=\sum_{l=0} \infty \frac{\Gamma(6 l+1) \Gamma(2 l+1)}{\Gamma^{5}(l+1) \Gamma(3 l+1)}\left(\frac{-1}{(12 \psi)^{6}}\right)^{l} .
$$

But according to the conjecture, Eq. (3.51) is the fundamental period for $\mathbb{P}_{(1,1,1,1,1,3)}^{5}[6,2]$. Indeed, it can be shown[36] that this manifold is birational to $\mathbb{P}_{(1,1,2,2,6)}^{4}[12]$. 


\section{Hypersurfaces with Two Parameters}

\subsection{Fermat hypersurfaces}

For Fermat hypersurfaces it is possible to derive some general results from the explicit form of the fundamental period given in (2.28). There are five known examples of Fermat threefolds with $b_{11}=2$, and they belong to the families

$$
\begin{gathered}
\mathbb{P}_{(1,1,2,2,2)}^{4}[8]_{-168}^{86,2}, \quad \mathbb{P}_{(1,1,2,2,6)}^{4}[12]_{-252}^{128,2}, \quad \mathbb{P}_{(1,4,2,2,3)}^{4}[12]_{-144}^{74,2} \\
\mathbb{P}_{(1,7,2,2,2)}^{4}[14]_{-240}^{122,2}, \quad \mathbb{P}_{(1,1,1,6,9)}^{4}[18]_{-540}^{272,2},
\end{gathered}
$$

respectively. As Fermat polynomials are invariant under transposition, their mirrors are described by $\{P=0\} / H$, for some appropriate group $H$, with $P$ taking the form

$$
P=\sum_{j=0}^{4} x_{j}^{d / k_{j}}-d \psi x_{0} x_{1} x_{2} x_{3} x_{4}-\frac{d}{q_{0}} \phi x_{0}^{q_{0}} x_{1}^{q_{1}} x_{2}^{q_{2}} x_{3}^{q_{3}} x_{4}^{q_{4}}
$$

Using the relations of the Jacobian ideal [31] we can show that in all cases $D \stackrel{\text { def }}{=} d / q_{0}$ is an integer and furthermore that

$$
\frac{q_{i} k_{i}}{q_{0}}= \begin{cases}0 & i \geq D \\ 1 & i<D\end{cases}
$$

For $\mathbb{P}_{(1,1,1,6,9)}^{4}, D=3$, and for the remaining examples, $D=2$.

From (2.31), with $Q=m t=m q_{0}$ and realizing that $t, s_{i} \in \mathbb{Z}$ for $p=1$, the fundamental period is given by

$$
\varpi_{0}(\psi, \phi)=\sum_{n=0}^{\infty} \sum_{m=0}^{\infty} \frac{\left(d n+q_{0} m\right) !(d \psi)^{-d n-q_{0} m}(-D \phi)^{m}}{m ! n ! \prod_{i=1}^{4}\left(k_{i} n+\frac{k_{i}}{d}\left(q_{0}-q_{i}\right) m\right) !}
$$

which converges for sufficiently large- $\psi$. Since $D$ is an integer, it is convenient to set $l=D n+m$ and sum over $l$ and $n$ to obtain the alternative expression

$$
\varpi_{0}(\psi, \phi)=\sum_{l=0}^{\infty} \frac{\left(q_{0} l\right) !(d \psi)^{-q_{0} l}(-1)^{l}}{l ! \prod_{i=1}^{4}\left(\frac{k_{i}}{d}\left(q_{0}-q_{i}\right) l\right) !} U_{l}(\phi)
$$

where

$$
U_{l}(\phi)=(D \phi)^{l} \sum_{n=0}^{\left[\frac{l}{D}\right]} \frac{l ! \prod_{i=1}^{4}\left(\frac{k_{i}}{d}\left(q_{0}-q_{i}\right) l\right) !(-D \phi)^{-D n}}{(l-D n) ! n ! \prod_{i=1}^{4}\left(\frac{k_{i} q_{i}}{q_{0}} n+\frac{k_{i}}{d}\left(q_{0}-q_{i}\right) l\right) !}
$$


$U_{l}(\phi)$ is a polynomial of degree $l$ but it can be extended to an hypergeometric function for complex values $\nu$ of $l$. For instance, when $D=2$ we find

$$
U_{\nu}(\phi)=(2 \phi)_{2}^{\nu} F_{1}\left(-\frac{\nu}{2}, \frac{1-\nu}{2} ; 1+\frac{\nu\left(k_{1}-1\right)}{2} ; \frac{1}{\phi^{2}}\right) \quad, \quad|\phi|>1
$$

where we have used (4.3).

$U_{\nu}(\phi)$ can be continued to the region $|\phi|<1$ by means of contour integrals or by using well known properties of the hypergeometric function. When $D=2$ the result is

$$
U_{\nu}(\phi)=\frac{e^{i \pi \nu / 2} \Gamma\left(1+\frac{\nu\left(k_{1}-1\right)}{2}\right)}{2 \Gamma(-\nu)} \sum_{m=0}^{\infty} \frac{e^{i \pi m / 2} \Gamma\left(\frac{m-\nu}{2}\right)(2 \phi)^{m}}{m ! \Gamma\left(1-\frac{m-\nu k_{1}}{2}\right)}
$$

which can in turn be written as a combination of hypergeometric functions around $\phi=0$.

Following the discussion in Section 2, the region of convergence for the fundamental period (4.4) can be found for the first two examples in (4.1)[7]. For example, for the first case we obtain that (4.4) converges in the region $\left|(\phi \pm 1) / 8 \psi^{4}\right|$. Unfortunately, the same analysis has proven more difficult for the other examples with the main problem being the calculation of the asymptotic behaviour of $U_{\nu}(\phi)$ as $\nu \rightarrow \infty$.

In order to obtain a basis of independent periods as explained in Section 2, we need to analytically continue $\varpi_{0}(\psi, \phi)$ to a small- $\psi$ region. Continuing the series given in (4.5) leads to an equivalent result by virtue of (4.8). Thus, when $D=2$, we find

$$
\varpi_{0}(\psi, \phi)=-\frac{2}{d} \sum_{n=1}^{\infty} \frac{\Gamma\left(\frac{2 n}{d}\right)(-d \psi)^{n} U_{-\frac{2 n}{d}}(\phi)}{\Gamma(n) \Gamma\left(1-\frac{n}{d}\left(k_{1}-1\right)\right) \Gamma\left(1-\frac{k_{2} n}{d}\right) \Gamma\left(1-\frac{k_{3} n}{d}\right) \Gamma\left(1-\frac{k_{4} n}{d}\right)}
$$

A basis of periods can be obtained by the action of the phase symmetry of the parameters as discussed in subsection 2.5. We define

$$
\varpi_{j}(\psi, \phi)=\varpi_{0}\left(\lambda^{j} \psi, \lambda^{j q_{0}} \phi\right) \quad, \quad j=0,1 \cdots, d-1
$$

where $\lambda^{d}=1$. Note that since Fermat polynomials are invariant under transposition, $\hat{d}=d$. Again, not all of the $\varpi_{j}$ are independent. In particular, the $\varpi_{j}$ satisfy

$$
\sum_{j=0}^{d-1} \varpi_{j}(\psi, \phi)=0
$$

Further relations among the $\varpi_{j}(\psi, \phi)$ arise from the fact that some terms in the series (4.9) are absent due to the $\Gamma$-functions in the denominator. The resulting constraints on the $\varpi_{j}$ depend on the specific values of the weights $k_{i}$. In all cases we find that as expected there are only six independent periods. 


\subsection{A non-Fermat example}

We turn now to an example which is interesting because it is not of Fermat type and it illustrates the important fact that the periods are most naturally written in terms of the weights of the mirror manifolds. This is not evident in Fermat examples precisely because the mirrors in these cases are quotients of the original manifold.

Consider the following quasihomogenous polynomial,

$$
P_{0}=x_{0}^{7}+x_{1}^{7} x_{3}+x_{3}^{3}+x_{2}^{7} x_{4}+x_{4}^{3} .
$$

$P_{0}=0$ defines a hypersurface $\mathcal{M}_{0}$ in a weighted projective space $\mathbb{P}_{(3,2,2,7,7)}^{4}[21]_{-78}^{50,11}$. As it is, the space of complex structures for this model is 50-dimensional and we wish to focus on a smaller subspace. To this end, we consider a 2-parameter deformation

$$
P_{\phi_{0}, \phi_{1}}=x_{0}^{7}+x_{1}^{7} x_{3}+x_{3}^{3}+x_{2}^{7} x_{4}+x_{4}^{3}-\phi_{0} x_{0} x_{1} x_{2} x_{3} x_{4}+\phi_{1} x_{0}^{3} x_{1}^{3} x_{2}^{3} .
$$

This 2-parameter family of polynomials is invariant under the symmetry

$$
H \stackrel{\text { def }}{=}\left(\mathbb{Z}_{21}: 12,2,0,7,0\right),
$$

and in fact is the most general subclass of such $\mathbb{P}_{(3,2,2,7,7)}^{4}[21]$ models; $P_{\phi_{0}, \phi_{1}}$ is the superpotential for the corresponding Landau-Ginzburg orbifolds. Note that $H$ is not the quantum symmetry

$$
Q_{\mathcal{M}}=\left(\mathbb{Z}_{21}: 3,2,2,7,7\right)
$$

although both $Q_{\mathcal{M}}$ and $H$ are isomorphic to $\mathbb{Z}_{21}$.

Related to this is the 2-parameter family $\mathcal{M}_{\phi_{0}, \phi_{1}} / H$, for which $\left(b_{2,1}, b_{1,1}\right)=$ $(2,95)$. Furthermore, the orbifold $\mathcal{M}_{0} / H$ is the mirror model of a hypersurface $\mathcal{W}$ in $\mathbb{P}_{(1,1,1,2,2)}^{4}[7]_{-186}^{95,2}$ for which the two Kähler variations are mirrored in $\phi_{0}, \phi_{1}$. This can then be used to study both the space of Kähler classes on $\mathcal{W}$ and the space of complex structures for $\mathcal{M} / H$. Let us also point out that the geometric symmetry of $\mathcal{M} / H$ is

$$
G_{\mathcal{M} / H}=\left(\mathbb{Z}_{7}: 6,0,0,0,0\right) \text {. }
$$

Now, $G_{\mathcal{M} / H}$ will leave $P_{\phi_{0}, \phi_{1}}$ invariant if also

$$
G_{\mathcal{M} / H}:\left(\phi_{0}, \phi_{1}\right) \longrightarrow\left(\lambda \phi_{0}, \lambda^{3} \phi_{1}\right), \quad \lambda^{7}=1 .
$$

Note that $G_{\mathcal{M} / H}$ is isomorphic to the quantum symmetry of $\mathcal{W}$, the mirror of $\mathcal{M} / H$.

Here, we are interested in obtaining explicit expressions for the periods as functions of $\phi_{0}, \phi_{1}$. From our general discussion of Section 2 we notice that using (2.8) and (2.10) we have

$$
q_{0}^{0}=q_{1}^{0}=q_{2}^{0}=3, \quad\left[a_{i j}\right]=\left[\begin{array}{ccccc}
7 & 0 & 0 & 0 & 0 \\
0 & 7 & 0 & 0 & 0 \\
0 & 0 & 7 & 0 & 0 \\
0 & 1 & 0 & 3 & 0 \\
0 & 0 & 1 & 0 & 3
\end{array}\right] .
$$


Furthermore, from the transpose of $a_{i j}$ we obtain the weights of the mirror manifold $\left(\hat{k}_{1}, \ldots, \hat{k}_{5}\right)=(1,1,1,2,2)$ and the degree of its defining polynomial $\hat{d}=7$. From Eqs. $(2.23),(2.24)$ and $(2.25)$ we have $n_{1}=n_{2}=n_{3}=r$ and $n_{4}=n_{5}=2 r+m$, and $s_{0,1,2}=0$, $s_{3,4}=1=p$ and $t=3$. This yields the fundamental period

$$
\varpi_{0}=\sum_{m, r=0}^{\infty} \frac{\Gamma(7 r+3 m+1)}{\Gamma^{3}(r+1) \Gamma^{2}(2 r+m+1)} \frac{1}{m !}\left(\frac{\phi_{1}}{\phi_{0}^{3}}\right)^{m} \phi_{0}^{-7 r} .
$$

Eq. (4.19) may be rewritten in a number of ways. Let us choose good coordinates for the large complex structure limit [36],

$$
X=\frac{\phi_{1}}{\phi_{0}^{3}} \quad ; \quad Y=\frac{1}{\phi_{0} \phi_{1}^{2}} .
$$

Then, by changing the summation by setting $l=m+2 r$, we find

$$
\varpi_{0}=\sum_{l=0}^{\infty} \frac{\Gamma(3 l+1)}{\Gamma^{3}(l+1)} X^{l} U_{l}(Y)
$$

where

$$
U_{l}(Y)=\frac{\Gamma(l+1)}{\Gamma(3 l+1)} \sum_{r=0}^{[l / 2]} \frac{\Gamma(r+3 l+1)}{\Gamma^{3}(r+1) \Gamma(-2 r+l+1)} Y^{r} .
$$

$U_{l}$ may be rewritten as a generalized hypergeometric function of type ${ }_{3} F_{2}$. In fact since the series terminates we have a polynomial. The period in the above form should prove useful in studying the monodromy of the periods around the singular curves in moduli space. This is however beyond the scope of the present analysis, in particular because of the lack of an asymptotic form for $U_{l}$ when $l \rightarrow \infty$, as discussed in Section 2.

The next step is to analytically continue $\varpi_{0}$ to small- $\phi_{0}$. From our general analysis in Section 2, we find

$$
\varpi_{0}\left(\phi_{0}, \phi_{1}\right)=-\frac{1}{7} \sum_{n=1}^{\infty} e^{6 i \pi n / 7} \frac{\phi_{0}^{n}}{\Gamma(n)} \sum_{m=0}^{\infty} \frac{e^{-3 i \pi m / 7} \Gamma\left(\frac{n+3 m}{7}\right)}{\Gamma^{2}\left(1-\frac{n+3 m}{7}\right) \Gamma^{2}\left(1-\frac{2 n-m}{7}\right)} \frac{\phi_{1}^{m}}{m !}
$$

The remaining periods are then obtained from (4.23) by acting with the modular group. Observe that terms with $(n+3 m)=0 \bmod 7$ are absent in (4.23) due to the $\Gamma\left(1-\frac{n+3 m}{7}\right)$ in the denominator, and so consider the action

$$
\mathcal{A}:\left(\phi_{0}, \phi_{1}\right) \longmapsto\left(\lambda^{j} \phi_{0}, \lambda^{3 j} \phi_{1}\right)
$$

and define

$$
\varpi_{j}\left(\phi_{0}, \phi_{1}\right) \stackrel{\text { def }}{=} \varpi_{0}\left(\lambda \phi_{0}, \lambda^{3} \phi_{1}\right), \quad j=0, \cdots, 6 .
$$


The $\varpi_{j}$ are subject to the single relation

$$
\sum_{j=0}^{6} \varpi_{j}=0 .
$$

Since $b_{3}=6$ for the 2-parameter model $\mathcal{M} / H$, these six linearly independent periods form a complete set of periods for the space of complex structures on $\mathcal{M} / H$ and also for the space of Kähler classes on $\mathcal{W}$.

The appearance of the $\Gamma\left(1-\frac{2 n-m}{7}\right)$ may prompt considering

$$
\mathcal{B}:\left(\phi_{0}, \phi_{1}\right) \longmapsto\left(\lambda^{2} \phi_{0}, \lambda^{-1} \phi_{1}\right),
$$

and define more periods using this action. However, in fact, $\mathcal{B}=\mathcal{A}^{2}$ (since $\lambda^{6}=\lambda^{-1}$ ) and no new periods or conditions ensue.

Consider now briefly the complementary components of the complete moduli space. To that end, we turn to the model $\mathcal{W} \in \mathbb{P}_{(1,1,1,2,2)}^{4}[7]$, defined by the transposed defining equation

$$
\hat{P}_{0}=y_{1}^{7}+y_{2}^{7}+y_{2} y_{4}^{3}+y_{3}^{7}+y_{3} y_{5}^{3},
$$

associated to the transposed matrix of exponents in (4.18). Besides the fundamental monomial $\hat{M}_{0}=y_{1} y_{2} y_{3} y_{4} y_{5}$, this model has 94 independent deformations. The quantum symmetry being $\left(\mathbb{Z}_{7}: 1,1,1,2,2\right)$, the geometric symmetry of $\mathcal{W}$ is

$$
G_{\mathcal{W}}=\left(\mathbb{Z}_{21}: 0,18,0,1,0\right) \times\left(\mathbb{Z}_{21}: 0,0,18,0,1\right)
$$

Note that $G_{\mathcal{W}}$ is isomorphic to $Q_{\mathcal{M} / H}$, the quantum symmetry of $\mathcal{M} / H$, as it should be since $\mathcal{W}$ and $\mathcal{M} / H$ are mirror models.

The techniques of Section 2 enable us to calculate the complex structure periods $\hat{\varpi}_{0}\left(\theta_{\beta}\right), \beta=0,1, \ldots, 94$ for the 95 -parameter family of models with

$$
\hat{P}_{\theta_{\beta}}=\hat{P}_{0}-\theta_{0} y_{1} y_{2} y_{3} y_{4} y_{5}+\sum_{\beta=1}^{94} \theta_{\beta} \hat{M}_{\beta}\left(y_{i}\right) .
$$

Once the small- $\theta_{0}$ expansion of $\hat{\varpi}_{0}$ is obtained, for example upon analytic continuation of the large- $\theta_{0}$ expansion as described above, other periods will be obtained through the action of the modular group. In the present case, the action of $G_{\mathcal{W}}$, defined in (4.27), is matched by the induced action $\mathcal{A}_{\mathcal{W}}: \theta_{0} \rightarrow \lambda^{2} \mu^{2} \theta_{0}$, etc., so as to make $P_{\theta_{\beta}}(y)$ invariant. Note that the action of $G_{\mathcal{W}}$ (and $\mathcal{A}_{\mathcal{W}}$ ) is rather more involved than the simple case in Eqs. (4.16)-(4.17), but is of the general form discussed in subsection 2.5. 


\subsection{The Picard-Fuchs equations}

The periods of $\Omega$ can also be determined as solutions of certain Picard-Fuchs differential equations. These equations can be derived from the defining polynomial $p$ by means of an algebraic geometry construction [4,37-40], originally due to Griffiths [41]. We have analyzed particular examples of the Fermat surfaces discussed above using the techniques explained in Refs. $[37,5]$ and found the linear system of partial differential equations satisfied by the periods.

With the benefit of hindsight, we can describe how the Picard-Fuchs equations follow from our fundamental period. For concreteness consider $\mathbb{P}_{(1,1,2,2,6)}^{4}[12]$ and define

$$
R(\psi, \phi)=\frac{1}{\psi} \varpi_{0}(\psi, \phi)
$$

$R(\psi, \phi)$ is given by a double series of the form

$$
R(\psi, \phi)=\sum_{n, m=0}^{\infty} A_{n, m} \psi^{n} \phi^{m}
$$

where the coefficients $A_{n, m}$ can be read off from (4.8) and (4.9). The equations satisfied by $R(\psi, \phi)$ are equivalent to recurrence relations verified by $A_{n, m}$. For instance, since $A_{n+q_{0}, m} / A_{n, m+1}$, with $q_{0}=6$, is a rational function of $n$ and $m$, this relation derives from a partial differential equation. Likewise, $A_{n, m+2} / A_{n, m}$ is a rational function and the corresponding equation reflects the singularity at $\phi^{2}=1$. The last equation is associated with the conifold type singularity that exists at $\left(864 \psi^{6}+\phi\right)^{2}=1$. 


\section{Other Important Generalizations}

In this section we show that the periods can be calculated even in the cases where there is no known mirror. We show also that the fundamental period can be obtained even when including non-polynomial deformations of the defining polynomial. In particular, in Ref. [27] it has been shown that some of the parameters associated to the twisted sectors in Landau-Ginzburg theories can be represented by certain non-polynomial deformations. We find however that it is necessary to introduce new contours associated to small values of the parameters.

\subsection{Manifolds with no known mirror}

It was mentioned in Section 2 that the Ansatz (2.8) for the form of the reference polynomial does not cover all non-degenerate models and that there are several classes which require more monomials than there are coordinates. We now wish to illustrate how the above analysis applies to such models.

Consider a model from the $\mathbb{P}_{(3,4,1,3,2)}^{4}[13]_{-114}^{62,5}$ class. A little experimentation shows that there is no non-degenerate polynomial consisting of five monomials in this class. This example therefore goes beyond the lists of Ref. [11] and in fact shows how to deal with all the remaining models found in Refs. [8,9].

In the case of $\mathbb{P}_{(3,4,1,3,2)}^{4}[13]$ it is enough to introduce an extra monomial to obtain a transverse polynomial. We will calculate then the period for the model

$$
\begin{aligned}
& P_{0}=x_{0}^{3} x_{1}+x_{1}^{3} x_{2}+x_{2}^{13}+x_{2} x_{3}^{4}+x_{3} x_{4}^{5}, \\
& P=P_{0}+\phi x_{1} x_{3}^{3}-\psi x_{0} x_{1} x_{2} x_{3} x_{4},
\end{aligned}
$$

where, as explained, the reference polynomial $P_{0}$ is degenerate. Proceeding as before, we obtain

$$
\begin{aligned}
& \varpi_{0}=\sum_{r, m=0}^{\infty} \frac{1}{\psi^{45 r}} \frac{\Gamma(45 r+15 m+1)}{\Gamma(15 r+5 m+1) \Gamma(10 r+2 m+1) \Gamma(2 r+m+1)} \\
& \times \frac{1}{\Gamma(9 r+1) \Gamma(9 r+3 m+1)} \frac{\left(\phi^{4} / \psi^{15}\right)^{m}}{(4 m) !} .
\end{aligned}
$$

Comparing with the general expression (2.28), we record (since the index $\alpha$ takes only one value, we drop it):

$$
\hat{d}=45, \quad \hat{k}_{i}=15,10,2,9,9, \quad p=4, \quad s_{i}=5,2,1,0,3, \quad t=15,
$$

and introduce

$$
\varphi \stackrel{\text { def }}{=} \phi^{4} / \psi^{15} .
$$


Note the coefficient of $r$ in $\Gamma(45 r+15 m+1): 45$ is indeed the degree of the transpose,

$$
\hat{P}_{0}=x_{0}^{3}+x_{0} x_{1}^{3}+x_{1} x_{2}^{13} x_{3}+x_{3}^{4} x_{4}+x_{4}^{5},
$$

of the reference polynomial $P_{0}$ in (5.1). This transposed polynomial (5.6) is also degenerate. Worse, there is no polynomial deformation which makes it transverse; the entire family $\mathbb{P}_{(15,10,2,9,9)}^{4}[45]$ consists of singular models! The non-degeneracy of the reference polynomial (superpotential) is important only from the Landau-Ginzburg point of view and, in fact, the present study may well be useful in providing a sensible interpretation to degenerate Landau-Ginzburg models. We hope to return to this important problem soon [42].

Following the analysis in Section 2, from (5.3) we write the Mellin-Barnes integral representation:

$$
\begin{aligned}
& \varpi_{0}=-\frac{1}{2 \pi i} \int_{\gamma} \mathrm{d} \rho e^{i \pi \rho} \psi^{-45 \rho} \frac{\pi}{\sin \pi \rho} \Gamma(45 \rho+1) \\
& \times \sum_{m=0}^{\infty} \frac{(45 \rho+1) 15 m}{\Gamma(15 \rho+5 m+1) \Gamma(10 \rho+2 m+1) \Gamma(2 \rho+m+1) \Gamma(9 \rho+1) \Gamma(9 \rho+3 m+1)} \frac{\varphi^{m}}{(4 m) !},
\end{aligned}
$$

or

$$
\begin{aligned}
\varpi_{0}=-\frac{1}{2 \pi i} \int_{\gamma} \mathrm{d} \rho e^{i \pi \rho} \psi^{-45 \rho} \frac{\pi}{\sin \pi \rho} & \Gamma(45 \rho+1) \\
& \times \frac{\mathcal{W}_{\rho}(\varphi)}{\Gamma(15 \rho+1) \Gamma(10 \rho+1) \Gamma(2 \rho+1) \Gamma^{2}(9 \rho+1)},
\end{aligned}
$$

where

$$
\mathcal{W}_{\rho}(\varphi) \stackrel{\text { def }}{=} \sum_{m=0}^{\infty} \frac{(45 \rho+1)_{15 m}}{(15 \rho+1)_{5 m}(10 \rho+1)_{2 m}(2 \rho+1)_{m}(9 \rho+1)_{3 m}} \frac{\varphi^{m}}{4^{4 m}\left(\frac{1}{4}\right)_{m}\left(\frac{2}{4}\right)_{m}\left(\frac{3}{4}\right)_{m} m !} .
$$

upon a little algebra, we obtain

$$
\begin{aligned}
& \mathcal{W}_{\rho}(\varphi)= \\
& { }_{8} F_{7}\left(\begin{array}{c}
3 \rho+\frac{1}{15}, 3 \rho+\frac{2}{15}, 3 \rho+\frac{4}{15}, 3 \rho+\frac{7}{15}, 3 \rho+\frac{8}{15}, 3 \rho+\frac{11}{15}, 3 \rho+\frac{13}{15}, 3 \rho+\frac{14}{15} \\
5 \rho+\frac{1}{2}, \quad 5 \rho+1, \quad 2 \rho+1, \quad 3 \rho+1, \quad \frac{1}{4}, \quad \frac{1}{2}, \quad \frac{3}{4}
\end{array} ;\left(\frac{3^{12} 5^{10}}{2^{2} 4^{4}} \varphi\right)\right) .
\end{aligned}
$$

As discussed in Section 2, the convergence of the integral in (5.8) depends on the asymptotic behaviour of $\mathcal{W}_{\rho}$ for $|\rho| \rightarrow \infty$ and ascertaining this is a difficult task [34]. Instead, we assume that the relevant integrals converge for suitably small values of $\varphi$ and proceed with the evaluation of the integral (5.8) by residues.

As usual, by closing the contour to the right, the (simple) poles of $1 / \sin \pi \rho$ at $\rho=$ $r=0,1,2, \ldots$ contribute and reproduce the small- $\psi$ expansion (5.3). 
Closing the contour to the left, the following poles are encircled:

1. $\rho=-1,-2,-3, \ldots$ from both $1 / \sin \pi \rho$ and $\Gamma(45 \rho+1)$,

2. $\rho=-\left(R+\frac{r}{45}\right), r=1,2, \ldots, 44$ and $R=0,1,2, \ldots$ from $\Gamma(45 \rho+1)$.

Now, in $(5.7)$, the zeros of $1 / \Gamma(9 \rho+1)$ include negative integers, and since $s_{4}=0$, the position of these zeros does not shift with growing $m$; think of these zeros as cancelling the poles from $1 / \sin \pi \rho$. It should also be clear from $(5.7)$, the integral- $\rho$ poles of $\Gamma(45 \rho+1)$ are completely cancelled by the combined zeros of $1 / \Gamma(10 \rho+2 m+1)$ and of $(45 \rho+1)_{15 m}$. Alternatively, from (5.3), the poles of $\Gamma(45 \rho+15 m+1)$ begin at $\rho=-(15 m+1) / 45$, while the zeros of $1 / \Gamma(10 \rho+2 m+1)$ begin at $\rho=-(2 m+1) / 10$, whence the integral- $\rho$ poles are all cancelled and for all $m=0,1, \ldots$

The integral in (5.8), with the contour closed to the left, may thus be evaluated by summing the (simple pole) residues located at $\rho=-\left(R+\frac{r}{45}\right)$, with $R=0,1,2, \ldots$ and $r=1,2, \ldots, 44$. Without further ado:

$$
\begin{aligned}
\varpi_{0}=\frac{\pi}{45} \sum_{r=1}^{44} \frac{e^{\frac{i \pi r}{45}}}{\sin \left(\frac{\pi r}{45}\right)}(-\psi)^{r} \sum_{R=0}^{\infty} \frac{(-\psi)^{45 R}}{\Gamma(45 R+r)} \\
\quad \times \frac{\mathcal{W}_{-\left(R+\frac{r}{45}\right)}(\varphi)}{\Gamma\left(1-15 R-\frac{r}{3}\right) \Gamma\left(1-10 R-\frac{10 r}{45}\right) \Gamma\left(1-2 R-\frac{2 r}{45}\right) \Gamma\left(1-9 R-\frac{r}{5}\right)},
\end{aligned}
$$

Note that we have not taken care explicitly of all cancellations, hence many of the terms in this expansion actually vanish.

For a cursory search for other periods ${ }^{8}$, rewrite (5.11) more explicitly as

$$
\begin{aligned}
& \varpi_{0}=\frac{\pi}{45} \sum_{r=1}^{44} \frac{e^{\frac{i \pi r}{45}}}{\sin \left(\frac{\pi r}{45}\right)}(-\psi)^{r} \sum_{R=0}^{\infty}(-\psi)^{45 R} \sum_{m=0}^{\infty} \frac{(-1)^{15 m}}{\Gamma(45 R+r-15 m)} \\
& \times \frac{\varphi^{m} /(4 m) !}{\Gamma\left(1+5 m-15 R-\frac{r}{3}\right) \Gamma\left(1+2 m-10 R-\frac{10 r}{45}\right) \Gamma\left(1+m-2 R-\frac{2 r}{45}\right) \Gamma\left(1+5 m-9 R-\frac{r}{5}\right)} .
\end{aligned}
$$

Recall that $\varphi=\phi^{4} \psi^{-15}$, so if $n$ is the exponent of $\psi$, we have $r=n+15 m$. From the $\Gamma$-functions in the denominator in (5.12) only the fractions provide non-trivial conditions and

$$
\begin{array}{rlrl}
r=n+15 m=0(\bmod 3) & :\left(\psi, \phi^{4}\right) & \mapsto\left(\lambda \psi, \phi^{4}\right), & \lambda^{3}=1, \\
10 r=10 n+15 m=0(\bmod 45) & :\left(\psi, \phi^{4}\right) \mapsto\left(\lambda^{10} \psi, \lambda^{15} \phi^{4}\right), & \lambda^{45}=1, \\
& \approx\left(\psi, \phi^{4}\right) \mapsto\left(\lambda^{2} \psi, \lambda^{3} \phi^{4}\right), & \lambda^{9}=1, \\
2 r=2 n+30 m=0(\bmod 45) & :\left(\psi, \phi^{4}\right) \mapsto\left(\lambda^{2} \psi, \lambda^{30} \phi^{4}\right), & \lambda^{45}=1, \\
r=n+15 m=0(\bmod 5) & :\left(\psi, \phi^{4}\right) \mapsto\left(\lambda \psi, \phi^{4}\right), & \lambda^{5}=1 .
\end{array}
$$

8 Eq. (5.2) does not include the full complement of deformations and we cannot hope to recover the $b_{3}=126$ periods. 
It is easy to check that all of these actions may be generated from

$$
\varpi_{j}\left(\psi, \phi^{4}\right) \stackrel{\text { def }}{=} \varpi_{0}\left(\lambda \psi, \lambda^{15} \phi^{4}\right), \quad \lambda^{45}=1 .
$$

These 45 periods however satisfy the relation $\sum_{j=1}^{45} \varpi_{j}=0$, so that at most 44 of them are independent.

\subsection{Twisted moduli}

Recall the complete intersection space (3.46)

$$
\mathcal{M} \in \mathbb{P}^{3}\left[\begin{array}{ll}
3 & 1 \\
\mathbb{P}^{2} & 3
\end{array}\right]_{-54}^{35,8}:\left\{\begin{array}{r}
p_{1,0}(x)=\sum_{i=0}^{3}\left(x_{i}\right)^{3}=0 \\
p_{2,0}(x, y)=\sum_{i=1}^{3} x_{i} y_{i}^{3}=0
\end{array}\right.
$$

for which we obtained the Kähler class fundamental period in subsection 3.3. However, we obtained the period just as a function of the two parameters corresponding to the Kähler classes of the two projective spaces. In fact, $b_{1,1}=8$ for $\mathcal{M}$ and so there are six more parameters.

It turns out that the additional six elements of $H^{1,1}(\mathcal{M})$ may be assigned explicit representatives, these however contain square-roots [27]. The six additional elements may be given as 'radical deformations' of the general form:

$$
\tilde{M}_{\alpha} \stackrel{\text { def }}{=} Q_{\alpha}\left(x_{i}\right) \sqrt{y_{1} y_{2} y_{3}}
$$

where $Q_{\alpha}\left(x_{i}\right)$ are six suitable quadrics in the $x_{i}$. Such elements cannot be added to either of the two defining equations in (5.19), as they have bi-degree $\left(2, \frac{3}{2}\right)$. However it is interesting that the complete intersection space (3.46) has an alternative description in terms of a single hypersurface, $\mathbb{P}_{(3,3,3,3,2,2,2)}^{6}[9]$, which also has a Landau-Ginzburg model associated to it [18]. The reference defining equation (superpotential) is then taken to be

$$
P_{0}(x, y)=p_{1,0}(x)+p_{2,0}(x, y)=x_{0}^{3}+\sum_{i=1}^{3}\left(x_{i}^{3}+x_{i} y_{i}^{3}\right)
$$

As the coordinates now have weights $k_{x}=3$ and $k_{y}=2$, the elements in (5.18) have the appropriate degree 9 and may appear as deformations of the reference polynomial:

$$
\begin{aligned}
P(x, y)=x_{0}^{3} & +\sum_{i=1}^{3}\left(x_{i}^{3}+x_{i} y_{i}^{3}\right)-\psi_{1} x_{1} x_{2} x_{3}-\psi_{2} x_{0} y_{1} y_{2} y_{3} \\
& +\sqrt{y_{1} y_{2} y_{3}} \sum_{i=1}^{3}\left(\alpha_{i} x_{i}^{2}+\beta_{i} x_{i+1} x_{i+2}\right)
\end{aligned}
$$


where the indices on $x_{i}$ are understood to be reduced modulo 3 .

Now, for the complete intersection space (3.46), it is natural to realize the periods as integrals of the form

$$
\int \frac{\mathrm{d}^{4} x \mathrm{~d}^{3} y}{p_{1}(x) p_{2}(x, y)},
$$

where $p_{1}(x)$ and $p_{2}(x, y)$ were defined in Eq. (3.47). However, relative to the representation (5.20), the periods are most naturally realised as

$$
\int \frac{\mathrm{d}^{4} x \mathrm{~d}^{3} y}{(P(x, y))^{2}}
$$

The previous procedure of expanding the period in inverse powers of the fundamental parameter is no longer applicable. The difficulty may be stated in a number of different ways. One is that there is no fundamental monomial for this case since the quantity $x_{0} x_{1} x_{2} x_{3} y_{1} y_{2} y_{3}$ does not have the correct degree and so may not be added to $P$. Related to this is the fact that attempts to define a fundamental contour in a manner analogous to previous cases fail owing to the fact that the 'fundamental contour' so defined is trivial in homology. These facts seem to be true generally for the generalised Calabi-Yau manifolds. Fortunately we are able to proceed by introducing contours analogous to those appropriate to small values of the fundamental parameters.

We proceed now to calculate a period from (5.22). To this end we take $x_{0}=1$ and let $\gamma_{i}$ be the contour in the $y_{i}$-plane that connects the points $\left(e^{\frac{2 \pi i}{3}} \infty, 0, \infty\right)$ and let $c_{i}$ be the contour in the $x_{i}$-plane that connects the points $(-\infty+i \epsilon, \infty+i \epsilon)$. Furthermore set $\Gamma=\gamma_{1} \times \gamma_{2} \times \gamma_{3}$ and $C=c_{1} \times c_{2} \times c_{3}$. The period (5.22) then becomes

$$
\varpi_{0} \stackrel{\text { def }}{=} \frac{1}{(2 \pi i)^{6}} \int_{\Gamma \times C} \frac{\mathrm{d}^{3} x \mathrm{~d}^{3} y}{P^{2}} .
$$

In order to perform the integrations we employ the identity

$$
\frac{1}{P^{2}}=-\int_{0}^{\infty} d s s e^{i s P}
$$

and separate $P$ from (5.20) into a reference polynomial $p_{0}$ and a remainder

$$
\begin{aligned}
P(x, y) & =p+\psi_{1} x_{1} x_{2} x_{3}+\psi_{2} y_{1} y_{2} y_{3}+\sqrt{y_{1} y_{2} y_{3}} \sum_{i=1}^{3}\left(\alpha_{i} x_{i}^{2}+\beta_{i} x_{i+1} x_{i+2}\right) \\
p & =1+\sum_{i=1}^{3}\left(x_{i}^{3}+x_{i} y_{i}^{3}\right) .
\end{aligned}
$$

On $\Gamma \times C$, the quantity $y_{i}^{3}$ is real and positive and the imaginary part of $x_{i}$ ensures the convergence of the integral in (5.24) and that of the $x$ - and $y$-integrals when we use (5.24) 
in (5.23). To proceed we expand out the dependence on the parameters. In order to avoid over-complicating the expressions that follow it is useful to adopt a multi-index notation such that if $x=\left(x_{1}, x_{2}, x_{3}\right)$ and $a=\left(a_{1}, a_{2}, a_{3}\right)$ then

$$
\begin{gathered}
x^{a} \stackrel{\text { def }}{=} x_{1}^{a_{1}} x_{2}^{a_{2}} x_{3}^{a_{3}}, \quad|a| \stackrel{\text { def }}{=} a_{1}+a_{2}+a_{3}, \\
a ! \stackrel{\text { def }}{=}\left(a_{1}\right) !\left(a_{2}\right) !\left(a_{3}\right) !
\end{gathered}
$$

and so on. With this understanding we find the following expression for $\varpi_{0}$ :

$$
\begin{aligned}
\varpi_{0}=\frac{i}{(2 \pi i)^{6}} & \sum_{n, m, a, b} \frac{\psi_{1}^{n} \psi_{2}^{m} \alpha^{a} \beta^{b}}{n ! m ! a ! b !} \int_{0}^{\infty} \mathrm{d} s e^{i s}(i s)^{n+m+|a|+|b|+1} \times \\
& \int_{C} \mathrm{~d}^{3} x e^{i s \sum_{i} x_{i}^{3}} x^{m+2 a+|b|-b} \int_{\Gamma} \mathrm{d}^{3} y e^{i s \sum_{j} x_{j} y_{j}^{3}} y^{n+\frac{1}{2}|a|+\frac{1}{2}|b|} .
\end{aligned}
$$

The $x$ - and $y$-integrals may be evaluated in virtue of the relations

$$
\int_{c} \mathrm{~d} \xi e^{i s \xi^{3}} \xi^{\sigma}=-\frac{2 \pi i e^{\frac{\pi i}{2}(\sigma+1)}}{3 s^{\frac{\sigma+1}{3}} \Gamma\left(\frac{2-\sigma}{3}\right)}, \quad \int_{\gamma} \mathrm{d} \eta e^{i s \xi \eta^{3}} \eta^{\rho}=-\frac{2 \pi i e^{\frac{\pi i}{2}(\rho+1)}}{3(s \xi)^{\frac{\rho+1}{3}} \Gamma\left(\frac{2-\rho}{3}\right)} .
$$

After a little algebra we arrive at the expression:

$$
\varpi_{0}=\frac{1}{3^{6}} \sum_{n, m, a, b} \frac{\psi_{1}^{n} \psi_{2}^{m} \alpha^{a} \beta^{b}}{n ! m ! a ! b !} \frac{e^{-\frac{\pi i}{3}(n+1)} \Gamma\left(\frac{n+1}{3}\right)}{\Gamma^{3}\left(\frac{2-n-\frac{1}{2}|a|-\frac{1}{2}|b|}{3}\right) \Gamma_{3}\left(\frac{2-m-2 a+b+\frac{1}{6}|a|-\frac{5}{6}|b|+\frac{n+1}{3}}{3}\right)} .
$$

The suffix on $\Gamma_{3}$ reminds us that, owing to the above multi-index conventions, this term corresponds to a product of three $\Gamma$-functions. We remark that if we set the twisted parameters to zero we recover the analytic continuation of the period defined by (3.49).

Clearly, there are many complete intersection spaces which do not have an alternate description in terms of a single hypersurface and/or a Landau-Ginzburg model, but this technique seems to present an important extension of the general discussion in Section 2 .

Indeed, such radicals also turn up in describing the complex structures. Borrowing from Ref. [26], we note that the complete intersection space

$$
\mathcal{M} \in \mathbb{P}^{4}\left[\begin{array}{ll}
4 & 1 \\
\mathbb{P}^{1} & 2
\end{array}\right]_{-168}^{86,2}:\left\{\begin{array}{cc}
p_{1,0}(x)=\sum_{i=0}^{4} x_{i}^{4} & =0 \\
p_{2,0}(x, y) & =x_{0} y_{0}^{2}+x_{1} y_{1}^{2}=0
\end{array}\right.
$$

has $b_{1,1}=2$. The Kähler class periods can be obtained easily as in Eqs. (3.47)-(3.49). The complex structure periods however pose somewhat of a problem since only 56 of the 86 
deformations may be realised as polynomial deformations of the defining equations (5.30). The 'missing' thirty deformations may however be realised as 'radical deformations' of the form $[27]$

$$
\tilde{M}_{\alpha}(x, y) \stackrel{\text { def }}{=} C_{\alpha}\left(x_{i}\right) \sqrt{x_{0} x_{1}},
$$

where $\left\{C\left(x_{i}\right)\right\}$ are thirty suitable monomials in $x_{i}$. Note moreover that this time the degree of these radical deformations is precisely right to be added to the first of the defining equations (5.30). In other words, the defining equations (5.30) may be deformed in the manner of Section 2, for example:

$$
\begin{aligned}
P_{1}(x) & =\sum_{i=0}^{4} x_{i}^{4}-\phi_{1} x_{0} x_{1} x_{2} x_{3}+\phi_{\alpha} M_{\alpha}(x)+\phi_{\alpha+56} C_{\alpha}\left(x_{i}\right) \sqrt{x_{0} x_{1}} \\
P_{2}(x, y) & =x_{0} y_{0}^{2}+x_{1} y_{1}^{2}-\phi_{2} x_{4} y_{0} y_{1}+\phi_{\beta} M_{\beta}(x, y)
\end{aligned}
$$

where $2<\alpha, \beta \leq 56$, so together with $x_{0} x_{1} x_{2} x_{3}$ and $x_{4} y_{0} y_{1}, M_{\alpha}(x)$ and $M_{\beta}(x, y)$ are the 56 conventional polynomial deformations. Therefore, the 86 parameters $\psi_{1}, \psi_{2}, \phi_{\alpha}$ then parametrize the full 86-dimensional space of complex structures. The corresponding periods may be realised as integrals of the type

$$
\int \frac{\mathrm{d}^{5} x \mathrm{~d}^{2} y}{P_{1}(x) P_{2}(x, y)},
$$

which may be calculated precisely as done in Sections 2 and 3.

It seems that the radical complex structure deformations tend to be of the correct degrees so they can be added as deformations of the defining equations of the original complete intersection model. By contrast, the radical Kähler class variations tend to occur in such a way that they cannot be added to the original defining equations of the complete intersections but may be used as a deformation of the superpotential of the corresponding Landau-Ginzburg orbifold, if such exists. In such cases, this superpotential invariably corresponds to the defining equation of a "generalized" Calabi-Yau hypersurface, where [43]

$$
d \stackrel{\text { def }}{=} \operatorname{deg}(P)=\frac{1}{Q} \sum_{i=0}^{N} k_{i}, \quad Q=2,3,
$$

holds instead of Eq. (2.1).

\subsection{A new look at the contours}

The purpose of this section is to repeat the calculation of periods for quintics in $\mathbb{P}^{4}$, using contours that are centered around the Fermat quintic. Of course, these results are well known but the idea is to illustrate the fact that these 'new' contours are the 
truly fundamental ones in the sense that there is generalization for them, not only to the class of models embedded in weighted $\mathbb{P}^{4}$, but to any Landau-Ginzburg theory whose superpotential defines a hypersurface in higher dimentional weighted projective spaces. We have already seen an example for the importance of the existence of these contours for the Schimmrigk manifold in subsection 5.2, where the 'fundamental' contour of Section 2 is trivial in homology.

Consider then, yet again, the model $\mathbb{P}^{4}[5]$. In the affine coordinate patch where $x_{5}=1$, the defining polynomial is

$$
P=1+\sum_{i=1}^{4} x_{i}^{5}-5 \psi x_{1} x_{2} x_{3} x_{4}
$$

We start by defining the periods as

$$
\begin{aligned}
\varpi_{j}(\psi) & \stackrel{\text { def }}{=} \psi\left(\frac{5}{2 \pi i}\right)^{4} \int_{\Gamma} \frac{\mathrm{d}^{4} x}{P}, \\
& =\psi\left(\frac{5}{2 \pi i}\right)^{4} \int_{\Gamma} \mathrm{d}^{4} x \int_{0}^{\infty} \mathrm{d} s e^{-s P}, \quad \Re e(P)>0
\end{aligned}
$$

Here, the poly-contours $\Gamma_{j}$ must be chosen so that the convergence criterion $\Re e(P)>0$ is satisfied. To this end, neglect temporarily the $\psi$-term and build the poly-contours $\Gamma_{j}=\prod_{i=1}^{4} \gamma_{i j}$ from pieces where

$$
x_{i}=\alpha^{k_{i}} \xi_{i}, \quad \alpha \stackrel{\text { def }}{=} e^{\frac{2 \pi i}{5}}, \quad k_{i} \in \mathbb{Z}, \quad 0 \leq \xi_{i}<\infty
$$

so that $x_{i}{ }^{5}>0$, and the integrals converge.

Therefore

$$
\begin{aligned}
\varpi_{0}(\psi) & =\psi\left(\frac{5}{2 \pi i}\right)^{4} \int_{0}^{\infty} \mathrm{d} s e^{-s} \int_{\Gamma} \mathrm{d}^{4} x e^{-s \sum_{i=1}^{4} x_{i}{ }^{5}} \sum_{n=0}^{\infty} \frac{(5 \psi)^{n}}{n !} s^{n} x_{1}{ }^{n} x_{2}{ }^{n} x_{3}{ }^{n} x_{4}{ }^{n} \\
& =\psi\left(\frac{5}{2 \pi i}\right)^{4} \sum_{n=0}^{\infty} \frac{(5 \psi)^{n}}{n !} \int_{0}^{\infty} \mathrm{d} s s^{n} e^{-s} \prod_{i=1}^{4} \int_{\gamma_{i, 0}} \mathrm{~d} x_{i} x_{i}{ }^{n} e^{-s x_{i}{ }^{5}} .
\end{aligned}
$$

The contours $\gamma_{i, 0}$ are chosen to connect $(\alpha \infty, 0, \infty)$, where $\alpha=e^{\frac{2 \pi i}{5}}$, so that

$$
\int_{\gamma_{0}}=\int_{\alpha \infty}^{0}+\int_{0}^{\infty}=\int_{0}^{\infty}-\int_{0}^{\alpha \infty}
$$

Next, use

$$
\int_{0}^{\alpha \infty} \mathrm{d} \xi \xi^{n} e^{-s \xi^{5}}=\alpha^{n+1} \int_{0}^{\infty} \mathrm{d} \xi \xi^{n} e^{-s \xi^{5}}=\frac{\alpha^{n+1}}{5} \frac{\Gamma\left(\frac{n+1}{5}\right)}{s^{\frac{n+1}{5}}}
$$


to obtain

$$
\begin{aligned}
\int_{\gamma_{0}} \mathrm{~d} \xi \xi^{n} e^{-s \xi^{5}} & =\frac{1}{5} \frac{\Gamma\left(\frac{n+1}{5}\right)}{s^{\frac{n+1}{5}}}\left(1-e^{\frac{2 \pi i}{5}(n+1)}\right) \\
& =\frac{e^{i \pi \frac{(n+1)}{5}}}{5} \frac{\Gamma\left(\frac{n+1}{5}\right)}{s^{\frac{n+1}{5}}}\left(-2 i \sin \left(\frac{n+1}{5} \pi\right)\right) \\
& =-\frac{2 \pi i}{5} \frac{e^{i \pi \frac{(n+1)}{5}}}{s^{\frac{n+1}{5}} \Gamma\left(1-\frac{n+1}{5}\right)}
\end{aligned}
$$

Thus,

$$
\begin{aligned}
\varpi_{0}(\psi) & =\psi\left(\frac{5}{2 \pi i}\right)^{4} \sum_{n=0}^{\infty} \frac{(5 \psi)^{n}}{n !} \int_{0}^{\infty} \mathrm{d} s s^{n} e^{-s}\left[-\frac{2 \pi i}{5} \frac{e^{i \pi \frac{(n+1)}{5}}}{s^{\frac{n+1}{5}} \Gamma\left(1-\frac{n+1}{5}\right)}\right]^{4} \\
& =\frac{1}{5} \sum_{n=0}^{\infty} \frac{(5 \psi)^{n+1}}{\Gamma(n+1)} \frac{e^{\frac{2 \pi i}{5} 2(n+1)}}{\Gamma^{4}\left(1-\frac{n+1}{5}\right)} \int_{0}^{\infty} \mathrm{d} s s^{\frac{n+1}{5}-1} e^{-s} \\
& =\frac{1}{5} \sum_{m=1}^{\infty} \frac{\left(5 \alpha^{2} \psi\right)^{m} \Gamma\left(\frac{m}{5}\right)}{\Gamma(m) \Gamma^{4}\left(1-\frac{m}{5}\right)}
\end{aligned}
$$

which exactly recovers the well known result.

Now note that rotating the contour $\gamma_{0}$ in the integral (5.41) counterclockwise in units of $\frac{2 \pi}{5}$, the result becomes

$$
\int_{\alpha^{j} \gamma_{0}} \mathrm{~d} \xi \xi^{n} e^{-s \xi^{5}}=-\frac{2 \pi i}{5} \frac{e^{i \pi \frac{(n+1)}{5}} \alpha^{j(n+1)}}{s^{\frac{n+1}{5}} \Gamma\left(1-\frac{n+1}{5}\right)} .
$$

Rotating only one of the four factor-contours in the poly-contour $\Gamma$ then produces

$$
\begin{aligned}
\varpi_{j}(\psi) & =\psi\left(\frac{5}{2 \pi i}\right)^{4} \sum_{n=0}^{\infty} \frac{(5 \psi)^{n}}{n !} \int_{0}^{\infty} \mathrm{d} s s^{n} e^{-s} \alpha^{j(n+1)}\left[-\frac{2 \pi i}{5} \frac{e^{i \pi \frac{(n+1)}{5}}}{s^{\frac{n+1}{5}} \Gamma\left(1-\frac{n+1}{5}\right)}\right]^{4}, \\
& =\varpi_{0}\left(\alpha^{j} \psi\right)
\end{aligned}
$$

which are the same five periods found originally.

Notably, we have not derived anything new. However, the periods have been now obtained by manifestly choosing different integration contours. Although we have not proven a priori that these different integration contours represent different cycles, this must be the case, since four of the five periods in Eq. (5.44) are linearly independent, which is the correct count. Moreover, the relation $\sum_{j=1}^{5} \varpi_{i}(\psi)=0$ acquires a convenient pictorial representation in terms of adding the contours ${ }^{9}$.

9 Of course, it is still important that every fifth term in the summation in Eq. (5.44) vanishes owing to the poles of the $\Gamma$-functions in the denominator. 


\section{Concluding Remarks}

We have described a method for computing the periods of Calabi-Yau models which is applicable to the majority of currently known Calabi-Yau manifolds and Landau-Ginzburg models. We recall that the calculation of the periods provides the basis for the explicit computation of the physically interesting quantities such as the physically normalized Yukawa couplings of the effective low energy theory and the threshold corrections to the gauge coupling constants [44] for $(2,2)$ models. Knowledge of the periods permits also the computation of the numbers of instantons of the corresponding Calabi-Yau manifold [3-7]. For a recent advance concerning instantons of genus one, see Ref. [45].

Therefore, the present work brings us significantly closer to having a complete control of the low energy effective action for any $(2,2)$ vacuum. It is illustrative to compare this with the conformal field theory (CFT) techniques used in the case of free theories and coset spaces. Even though the CFT is well known in those cases, the calculation of the effective couplings is always restricted to particular points (Gepner-Kazama-Suzuki models) or a subregion (orbifolds) of the whole moduli space of the given $(2,2)$ model. Remarkably, the geometrical approach used here has now achieved the same level of calculability as in the exact CFT's cases, even though the underlying CFT's are known explicitly only for special subregions or particular points of the moduli space. These achievements are due to the methods developed during the last few years using topological and algebrogeometrical techniques as well as special geometry and mirror symmetry. In addition, this method has the advantage that the dependence of the couplings on the whole moduli space is considered, whence the structure of the full modular group can be obtained. An illustrative example to compare both approaches is the mirror of the $Z_{3}$ orbifold [46]. In this case, the geometrical and the CFT methods give identical results for the relevant quantities. However, the explicit dependence of the couplings on the twisted moduli of the corresponding orbifold model may now be obtained using geometrical techniqueswhich goes beyond the common CFT practice. It would be interesting to obtain a similar level of computability for all couplings (including the $E_{6} 1$ 's) and eventually extend these techniques to $(0,2)$ models, where the geometrical techniques are far less well developed.

In principle the procedure presented here may be extended to other cases that were not considered, such as complete intersection spaces in products of weighted projective spaces [47] or more general Landau-Ginzburg models that do not have a standard CalabiYau interpretation [46]. Also, the methods of Sections 2 and 3 cover only the dependence of the Kähler class periods of CICY manifolds on the parameters corresponding to the Kähler classes of the embedding space or the mirror model. This appears to be suitably generalised by the inclusion of non-polynomial deformations and the alternate expansion method of Section 4. Another example of this type concerns the mirror of the $Z_{3}$ orbifold [46]. 
Finally, even though we can write the fundamental period for very large classes of models, the explicit determination of the full set of periods and the identification of the integral symplectic basis requires an understanding of the singularity structure of each moduli space and, at present, this has to be done model by model. Similar remarks apply also to the determination of the modular group since this is also largely determined by the singularity structure of the moduli space.

Acknowledgments: We are indebted to Paul Green and Sheldon Katz for discussions and corrections. This work was supported by the American-Scandinavian Foundation, the Fulbright Program, the NSF grants PHY 8904035 and PHY 9009850, the Robert A. Welch foundation and the Swiss National Science Foundation. P.B., X.D. and F.Q. would like to thank the Theory Division at CERN for hospitality and P.C. and X.D. wish to acknowledge the hospitality of the Institute for Theoretical Physics in Santa Barbara, California, where some of this work was done. A.F. thanks the Institut de Physique of the Université de Neuchâtel and the ICTP, Trieste for hospitality while part of this research was done as well as the Centro Científico IBM-Venezuela for the use of its facilities. 


\section{References}

1. A. Strominger, Commun. Math. Phys. 133 (1990) 163.

2. P. Candelas and X. C. de la Ossa, Nucl. Phys. B355 (1991) 455.

3. P. Candelas, X. de la Ossa, P. Green and L. Parkes, Nucl. Phys. B359 (1991) 21.

4. D. Morrison, "Picard-Fuchs Equations and Mirror Maps for Hypersurfaces", in Essays on Mirror Symmetry, ed. S.-T. Yau (Intl. Press, Hong Kong, 1992).

5. A. Font, Nucl. Phys. B391 (1993) 358.

6. A. Klemm and S. Theisen, Nucl. Phys. B389 (1993) 153.

7. P. Candelas, X. de la Ossa, A. Font, S. Katz and D. Morrison, in preparation.

8. M. Kreuzer and H. Skarke, Nucl. Phys. B388 (1992) 113.

9. M. Kreuzer and H. Skarke, Commun. Math. Phys. 150 (1992) 137.

10. A. Klemm and R. Schimmrigk, "Landau-Ginzburg Vacua", CERN preprint CERNTH-6459/92, Universität Heidelberg report HD-THEP-92-13.

11. P. Berglund and T. Hübsch, Nucl. Phys. B393 (1993) 377, also in Essays on Mirror Manifolds, p.388, ed. S.-T. Yau, (Intl. Press, Hong Kong, 1992).

12. B.R. Greene and M.R. Plesser: "Mirror Manifolds, A Brief Review and Progress Report", Cornell and Yale University preprints CLNS 91-1109, YCTP-P32-91.

13. T. Hübsch, Commun. Math. Phys. 108 (1987) 291.

14. P. Green and T. Hübsch, Commun. Math. Phys. 109 (1987) 99.

15. P. Candelas, A.M. Dale, C. A. Lütken and R. Schimmrigk, Nucl. Phys. B298 (1988) 493.

16. T. Hübsch: Calabi-Yau Manifolds-A Bestiary for Physicists (World Scientific, Singapore, 1992).

17. S.-T. Yau: in Proc. of Symposium on Anomalies, Geometry, Topology, pp.395, esp. the Appendix, by G. Tian and S.-T. Yau, pp.402-405, eds. W.A. Bardeen and A.R. White (World Scientific, Singapore, 1985).

18. B.R. Greene, C. Vafa and N.P. Warner, Nucl. Phys. B324 (1989) 371.

19. T. Hübsch, Class. Quantum Grav. 8 (1991) L31.

20. P. Berglund, B.R. Greene and T. Hübsch, Mod. Phys. Lett. A7 (1992) 1885.

21. A. Libgober and J. Teitelbaum, "Lines on Calabi-Yau Complete Intersections, Mirror Symmetry and Picard Fuchs Equations", University of Illinois Report (1992).

22. V. Batyrev, Duke Math. J. 69 (1993) 349.

23. R. Schimmrigk: Phys. Lett. 193B (1987) 175.

24. V. Batyrev and D. Van Straten, "Generalized Hypergeometric Functions and Rational Curves on Calabi-Yau Complete Intersections in Toric Varieties", Universität Essen report in preparation.

25. T. Hübsch and S.-T. Yau, Mod. Phys. Lett. A7 (1992) 3277. 
26. P. Green and T. Hübsch, Commun. Math. Phys. 113 (1987) 505.

27. P. Berglund and T. Hübsch, "Couplings for Compactification", Howard University preprint HUPAPP-93/2, Nucl. Phys. B (in press).

28. M. Lynker and R. Schimmrigk, Phys. Lett. 249B(1990) 237.

29. B.R. Greene and R. Plesser, in Essays on Mirror Symmetry, p.1, ed. S.-T. Yau (Intl. Press, Hong Kong, 1992).

30. M. Atiyah, R. Bott and L. Gårding, Acta Math. 131 (1973) 145.

31. P. Candelas, Nucl. Phys. B298 (1988) 458.

32. A. Erdélyi, F. Oberhettinger, W. Magnus and F. G. Tricomi, Higher Transcendental Functions, 3 Vols., (McGraw-Hill, New York, 1953).

33. P. Berglund and S. Katz, in preparation.

34. Y.L. Luke, The Special Functions and Their Approximations, 2 Vols., (Academic Press, New York, 1969).

35. A. Klemm and Theisen: "Mirror Maps and Instanton Sums for Complete Intersections in Weighted Projective Space", Munich University preprint LMU-TPW-93-08.

36. S. Katz, private communication.

37. A. C. Cadavid and S. Ferrara, Phys. Lett. 267B (1991) 193.

38. B. Blok and A. Varchenko, Int. J. Mod. Phys. A7, (1992) 1467.

39. W. Lerche, D. J. Smit and N. P. Warner, Nucl. Phys. B372 (1992) 87.

40. A. Ceresole, R. D'Auria, S. Ferrara, W. Lerche and J. Louis, Int. J. Mod. Phys.A8 (1993) 79.

41. P. Griffiths, Ann. Math. 90 (1969) 460.

42. P. Candelas, X. C. de la Ossa and S. Katz, work in progress

43. R. Schimmrigk: "Critical Superstring Vacua from Noncritical Manifolds: A Novel Framework", Universität Heidelberg report HD-THEP-92-29.

44. L. Dixon, Talk Presented at the Mirror Symmetry Workshop, MSRI (1991), unpublished.

45. M. Bershadsky, S. Cecotti, H. Ooguri and C. Vafa, "Holomorphic Anomalies in Topological Field Theories", Harvard University preprint HUTP-93/A008.

46. P. Candelas, E. Derrick and L. Parkes, "Generalized Calabi-Yau Manifolds and the Mirror of a Rigid Manifold", CERN preprint CERN-TH.6831/93 (1993).

47. B. Greene, S.-S. Roan and S.-T. Yau, Commun. Math. Phys. 142 (1991) 245. 